\title{
COHOMOLOGY OF STANDARD MODULES ON PARTIAL FLAG VARIETIES
}

\begin{abstract}
S. N. KITCHEN
Abstract. Cohomological induction gives an algebraic method for constructing representations of a real reductive Lie group $G$ from irreducible representations of reductive subgroups. Beilinson-Bernstein localization alternatively gives a geometric method for constructing Harish-Chandra modules for $G$ from certain representations of a Cartan subgroup. The duality theorem of Hecht, Miličić, Schmid and Wolf establishes a relationship between modules cohomologically induced from minimal parabolics and the cohomology of the $\mathscr{D}$-modules on the complex flag variety for $G$ determined by the BeilinsonBernstein construction. The main results of this paper give a generalization of the duality theorem to partial flag varieties, which recovers cohomologically induced modules arising from nonminimal parabolics.
\end{abstract}

\section{INTRODUCTION}

The objective of this paper is to extend the duality theorem of [HMSW] to partial flag varieties. For $G_{\mathbb{R}}$ a real reductive Lie group and $(\mathfrak{g}, K)$ its complex HarishChandra pair, the main difference between the geometry of $K$-orbits on the full flag variety of $\mathfrak{g}$ and on its partial flag varieties is that the orbits are not necessarily affinely embedded in the case of partial flag varieties, whereas they are for the full flag variety. The affineness of the embedding of $K$-orbits in the full flag variety of $\mathfrak{g}$ was used in an essential way in [HMSW]. Motivated by the derived equivariant constructions of $[\mathrm{P}$ and $[\mathrm{MP}$, we define analogous geometric constructions which allow us to prove our main result using derived category techniques, which take into account the failure of affineness of $K$-orbit embeddings.

1.1. Main Theorem. Before stating our main result, we first recall the duality theorem of [HMSW]. As above, let $G_{\mathbb{R}}$ denote a real reductive Lie group, to which we associate its complex Harish-Chandra pair $(\mathfrak{g}, K)$ and abstract Cartan triple $\left(\mathfrak{h}, \Sigma, \Sigma^{+}\right)$. On the full flag variety $X$ of $\mathfrak{g}$, let $Q$ be a $K$-orbit and $\tau$ an irreducible connection on $Q$. Such connections are parameterized by the irreducible representations of the stabilizer $S_{x}$ of an arbitrary point $x \in Q$. There is a twisted sheaf of differential operators (tdo) $\mathscr{D}_{\lambda}$ on $X$ for every $\lambda \in \mathfrak{h}^{*}$. We say the connection $\tau$ and the parameter $\lambda$ are compatible if the differential of the representation of $S_{x}$ determining $\tau$ is a direct sum of copies of $\lambda+\rho$ (restricted to the Lie algebra of $S_{x}$ ), where $\rho$ is the half-sum of positive roots. The $\mathscr{D}_{\lambda}$-modules on $X$ have cohomology groups which are Harish-Chandra modules with infinitesimal character $[\lambda] \in \mathfrak{h}^{*} / \mathcal{W}$, where $\mathcal{W}$ is the Weyl group associated to the data $\left(\mathfrak{h}, \Sigma, \Sigma^{+}\right)$. When $\tau$ and $\lambda$ are compatible, we define the standard module on $X$ corresponding to the pair $(\tau, \lambda)$ to

Received by the editors February 7, 2011 and, in revised form, January 20, 2012 and February 24,2012 .

2010 Mathematics Subject Classification. Primary 22-xx.

(c) 2012 American Mathematical Society 
be the $\mathscr{D}_{\lambda}$-module direct image $i_{+} \tau$ of $\tau$ along the inclusion $i: Q \rightarrow X$. Recall for $V$ a $(\mathfrak{b}, L)$-module with $\mathfrak{b} \subset \mathfrak{g}$ a Borel subalgebra and $L$ a subgroup of $K$, we induce $V$ to a $(\mathfrak{g}, L)$-module by taking the tensor product $\operatorname{ind}_{\mathfrak{b}, L}^{\mathfrak{g}, L}(V)=\mathcal{U}(\mathfrak{g}) \otimes_{\mathcal{U}(\mathfrak{b})} V$. Let $T_{x}$ denote the geometric fiber functor, which is the pull-back to the point $x$ together with a restriction of the group action to the stabilizer of $x$.

We state the main theorem of [HMSW not in its original form as a duality statement, but instead without contragredients so that it takes a form similar to the natural formulation of our main result.

Theorem 1.1 ([HMSW], Theorem 4.3). Let $x \in Q$ be any point, let $B_{x}$ be its stabilizer in $G$, and let $\mathfrak{b}_{x}$ be the Lie algebra of $B_{x}$. Put $\mathfrak{n}_{x}=\left[\mathfrak{b}_{x}, \mathfrak{b}_{x}\right]$ and let $\overline{\mathfrak{n}}_{x}$ be its opposite in $\mathfrak{g}$. Then for all $p \in \mathbb{Z}$, we have

$$
\mathrm{H}^{p}\left(X, i_{+} \tau\right) \simeq \mathbf{R}^{d_{Q}+p} \Gamma_{K, B_{x} \cap K}\left(\operatorname{ind}_{\mathfrak{b}_{x}, B_{x} \cap K}^{\mathfrak{g}, B_{x} \cap K}\left(T_{x} \tau \otimes \wedge^{\mathrm{top}} \overline{\mathfrak{n}}_{x}\right)\right)
$$

as $\mathcal{U}(\mathfrak{g})$-modules with infinitesimal character $[\lambda] \in \mathfrak{h}^{*}$.

For an infinitesimal character $\chi$, we, hereafter, use $\mathcal{U}_{\chi}$ to denote the quotient of $\mathcal{U}(\mathfrak{g})$ by the ideal determined by $\chi$ in the usual way, so that we may speak of $\mathcal{U}_{\chi}$-modules rather than " $\mathcal{U}(\mathfrak{g})$-modules with infinitesimal character $\chi$. "

Theorem 1.1 shows that the sheaf cohomology groups of standard $K$-equivariant $\mathscr{D}_{\lambda}$-modules on the full flag variety $X$ are isomorphic to cohomologically induced modules (specifically, modules which are induced from Borels). Our main result is the analogous identification of the cohomology of standard $\mathscr{D}_{\lambda}$-modules on a partial flag variety $X_{\theta}$, where $\theta$ is a subset of simple roots, with Harish-Chandra modules cohomologically induced from parabolics of type $\theta$.

Unfortunately, Theorem 1.1 fails to generalize immediately to partial flag varieties because the direct image functor $i_{+}$is not necessarily exact for the inclusion of a non-affinely embedded $K$-orbit $Q$ in $X_{\theta}$. So, the direct image $i_{+} \tau$ may be a complex of $\mathscr{D}_{\lambda}$-modules rather than a single sheaf. Theorem 1.2 below is an extension of Theorem 1.1 which incorporates the possible failure of exactness of $i_{+}$. Theorem 1.1 can be recovered as an immediate corollary. Let $p: X \rightarrow X_{\theta}$ be the natural projection from the full flag variety to $X_{\theta}$. Let $\rho_{\theta}$ be the half-sum of roots in the additive closure of $\theta$ in $\Sigma^{+}$and define $\rho_{n}=\rho-\rho_{\theta}$. There is a canonical subspace $\mathfrak{h}_{\theta}^{*} \subset \mathfrak{h}^{*}$ which parameterizes the homogeneous tdos on $X_{\theta}$. In particular, the element $\rho_{n}$ is in $\mathfrak{h}_{\theta}^{*}$ and takes over the role of $\rho$ in the definition of compatibility between connections $\tau$ and twists $\lambda$ in partial flag varieties. Our main theorem is then:

Theorem 1.2 (Main Theorem). Let $\mathscr{D}_{\lambda}$ be a homogeneous tdo on $X_{\theta}$ and let $\tau$ be a connection on a $K$-orbit $Q$ compatible with $\lambda$. For $x \in Q$, let $\mathfrak{p}_{x}$ be the corresponding parabolic in $\mathfrak{g}$, let $\mathfrak{n}_{x}$ be the nilradical of $\mathfrak{p}_{x}$, and let $S_{x}$ be the stabilizer of $x$ in $K$. Then, there is an isomorphism

$$
\mathbf{R} \Gamma\left(X, p^{\circ} i_{+} \tau\right) \simeq \Gamma_{K, S_{x}}^{\text {equi }}\left(\operatorname{ind}_{\mathfrak{p}_{x}, S_{x}}^{\mathfrak{g}, S_{x}}\left(T_{x} \tau \otimes \wedge^{\mathrm{top}} \overline{\mathfrak{n}}_{x}\right)\right)\left[d_{Q}\right]
$$

in $\mathrm{D}^{\mathrm{b}}\left(\mathcal{U}_{\left[\lambda-\rho_{\theta}\right]}, K\right)$, where $d_{Q}$ is the dimension of $Q$. Upon taking cohomology, there is a convergent spectral sequence

$$
\mathbf{R}^{p} \Gamma\left(X, p^{\circ} \mathbf{R}^{q} i_{+} \tau\right) \Longrightarrow \mathbf{R}^{d_{Q}+p+q} \Gamma_{K, S_{x}}\left(\operatorname{ind}_{\mathfrak{p}_{x}, S_{x}}^{\mathfrak{g}, S_{x}}\left(T_{x} \tau \otimes \wedge^{\text {top }} \overline{\mathfrak{n}}_{x}\right)\right)
$$

In this theorem, the category $\mathrm{D}^{\mathrm{b}}\left(\mathcal{U}_{\chi}, K\right)$ is the equivariant bounded derived category of Harish-Chandra modules with infinitesimal character $\chi$ and $\Gamma_{K, S}^{\text {equi }}$ is the 
equivariant Zuckerman functor introduced in $\S 3$. Naturally, the functor $\mathbf{R} \Gamma_{K, S}$ is the usual (derived) Zuckerman functor.

The spectral sequence (2) collapses in special cases, such as when $X_{\theta}$ is the full flag variety, but in general Theorem 1.2 is the closest we get to a direct generalization of Theorem 1.1. However, the convergence of (2) is sufficient for applications to composition series computations in the Grothendieck group of $\mathrm{D}^{\mathrm{b}}\left(\mathcal{U}_{\chi}, K\right)$.

The idea behind the proof of Theorem 1.2 is that the standard sheaf $i_{+} \tau$ is determined entirely by the geometric fiber $T_{x} \tau$ at a point $x \in Q$. We make this precise by constructing an essential inverse to the functor $T_{x}$. In this construction we introduce the geometric Zuckerman functor $\Gamma_{K, S}^{\text {geo }}$. The isomorphism (1) follows from the commutivity properties of $\Gamma_{K, S}^{\text {geo }}$, together with Theorem 1.3 below, which allows us to identify the $\mathcal{U}(\mathfrak{g})$-module structure on the sheaf cohomology in Theorem 1.2 .

Theorem 1.3 (Embedding Theorem). The inverse image functor $p^{\circ}: \mathcal{M}\left(\mathscr{D}_{\lambda}\right) \rightarrow$ $\mathcal{M}\left(\mathscr{D}_{\lambda}^{p}\right)$ is fully faithful for all $\lambda$, and for $\lambda$ anti-dominant, we have $\Gamma \circ p^{\circ}=p^{*} \circ \Gamma$, where $p^{*}: \mathcal{M}\left(\Gamma\left(\mathscr{D}_{\lambda}\right)\right) \rightarrow \mathcal{M}\left(\Gamma\left(\mathscr{D}_{\lambda}^{p}\right)\right)$ is the usual pull-back of modules induced by the natural map $\Gamma\left(\mathscr{D}_{\lambda}^{p}\right) \rightarrow \Gamma\left(\mathscr{D}_{\lambda}\right)$.

1.2. Contents of paper. In $\S \S 2$ and 3, we review twisted differential operators on homogeneous spaces and the construction of the equivariant Zuckerman functor $\Gamma_{K, S}^{\text {equi }}$ of $\left[\mathrm{P}\right.$. The functor $\Gamma_{K, S}^{\text {equi }}$ is the generalization of the usual derived Zuckerman functor to categories of derived equivariant complexes. In $[\mathrm{P}$, Pandžić proves that by taking cohomology of $\Gamma_{K, S}^{\text {equi }}$ we recover the usual Zuckerman functors. That is, for all $p$ we have

$$
\mathrm{H}^{p}\left(\Gamma_{K, T}^{\mathrm{equi}} V^{\bullet}\right)=\mathbf{R}^{p} \Gamma_{K, T}\left(V^{\bullet}\right) .
$$

Section 4 is the technical heart of the paper where we introduce the derived equivariant category of Harish-Chandra sheaves, define the geometric Zuckerman functor $\Gamma_{K, S}^{\mathrm{geo}}$ (which is the localization of $\Gamma_{K, S}^{\text {equi }}$ ), and prove that $\Gamma_{K, S}^{\mathrm{geo}}$ has sundry properties that will be used in the proof of the Theorem 1.2. In the final section, we prove Theorems 1.2 and 1.3 and end the paper with a brief reformulation of Theorem 1.2 as a duality statement.

\section{TWISTED SHEAVES OF DIFFERENTIAL OPERATORS}

In this section, we introduce our notation for the direct and inverse image of $\mathscr{D}$-modules, where $\mathscr{D}$ is a twisted sheaf of differential operators. Additionally, we give classification results for homogeneous sheaves of twisted differential operators on generalized flag varieties. We learned much of the material from Miličić's unpublished works [M1] and [M2].

2.1. Definitions. We will always use $\mathscr{D}_{X}$ to denote the sheaf of differential operators on a smooth complex algebraic variety $X$ and more generally $\mathscr{D}$ for a twisted sheaf of differential operators (tdo); that is, a sheaf of $\mathscr{O}_{X}$-algebras locally isomorphic to $\mathscr{D}_{X}$. Let $\mathcal{M}(\mathscr{D})$ denote the category of left $\mathscr{D}$-modules and $\mathrm{D}^{\mathrm{b}}(\mathscr{D})$ the corresponding bounded derived category. For right $\mathscr{D}$-modules we write $\mathcal{M}(\mathscr{D})_{\mathrm{r}}$ and $\mathrm{D}^{\mathrm{b}}(\mathscr{D})_{\mathrm{r}}$, respectively.

Fix a smooth map $f: Y \rightarrow X$ between smooth varieties and define $\mathscr{D}^{f}$ to be the sheaf of differential endomorphisms of the left $\mathscr{O}_{Y^{-}}$, right $f^{-1} \mathscr{D}$-module $\mathscr{D}_{Y \rightarrow X}=f^{*} \mathscr{D}$. The sheaf of operators $\mathscr{D}^{f}$ is itself a tdo on $Y$. In the trivial 
example, we have $\mathscr{D}=\mathscr{D}_{X}$ and $\mathscr{D}^{f}=\mathscr{D}_{Y}$ for any $f$. For maps $f: X \rightarrow Y$ and $g: Y \rightarrow Z$ and a tdo $\mathscr{D}$ on $Z$, we have $\left(\mathscr{D}^{g}\right)^{f} \simeq \mathscr{D}^{g \circ f}$.

2.2. Inverse image. Let $f: Y \rightarrow X$ and $\mathscr{D}$ be as in the above section. We denote the inverse image functor from $\mathcal{M}(\mathscr{D})$ to $\mathcal{M}\left(\mathscr{D}^{f}\right)$ by $f^{\circ}$. It is defined as

$$
f^{\circ}(-):=\mathscr{D}_{Y \rightarrow X} \otimes_{f^{-1} \mathscr{D}} f^{-1}(-) .
$$

Here $f^{-1}$ is the usual sheaf inverse image. The functor $f^{\circ}$ is right exact, exact when $f$ is flat, and has finite left cohomological dimension.

The category $\mathcal{M}(\mathscr{D})$ has enough projectives, and so the derived inverse image functor

$$
\mathbf{L} f^{\circ}: \mathrm{D}^{\mathrm{b}}(\mathscr{D}) \rightarrow \mathrm{D}^{\mathrm{b}}\left(\mathscr{D}^{f}\right)
$$

exists. In [B], Borel defines the functor

$$
f^{!}:=\mathbf{L} f^{\circ}\left[d_{Y / X}\right]: \mathrm{D}^{\mathrm{b}}(\mathscr{D}) \rightarrow \mathrm{D}^{\mathrm{b}}\left(\mathscr{D}^{f}\right),
$$

where $d_{Y / X}=\operatorname{dim} Y-\operatorname{dim} X$. Introducing the shift by $d_{Y / X}$ guarantees the functor $f^{!}$behaves well with respect to Verdier duality.

2.3. Direct image. Again let $f: Y \rightarrow X$ be as in $\$ 2.1$ and let $\mathscr{D}$ be a tdo on $X$. We will define the direct image functor $f_{+}$, then examine this functor for $f$ a surjective submersion. The opposite sheaf $\mathscr{D}^{\circ}$ of any tdo $\mathscr{D}$ is again a tdo M2, Prop. 11]. There is an isomorphism of categories $\mathcal{M}\left(\mathscr{D}^{\circ}\right)_{\mathrm{r}} \simeq \mathcal{M}(\mathscr{D})$, which is the identity on objects. Let $\omega_{Y / X}$ denote the relative canonical bundle for $f$.

Definition 2.1. Up to conjugation by the isomorphism $\mathcal{M}(\mathscr{D}) \simeq \mathcal{M}\left(\mathscr{D}^{\circ}\right)_{\mathrm{r}}$, the direct image functor $f_{+}: \mathrm{D}^{b}\left(\mathscr{D}^{f}\right) \rightarrow \mathrm{D}^{b}(\mathscr{D})$ is defined by

$$
f_{+}(-)=\mathbf{R} f_{*}\left(-\otimes \omega_{Y / X} \otimes_{(\mathscr{D} \circ)^{f}}^{\mathbf{L}} \mathscr{D}_{Y \rightarrow X}\right) .
$$

This definition is the translation to left $\mathscr{D}$-modules of the usual construction:

$$
f_{+}: \mathrm{D}^{\mathrm{b}}\left(\mathscr{D}^{f}\right)_{\mathrm{r}} \rightarrow \mathrm{D}^{\mathrm{b}}(\mathscr{D})_{\mathrm{r}}, \quad f_{+}(-)=\mathbf{R} f_{*}\left(-\otimes_{\mathscr{D}^{f}}^{\mathbf{L}} \mathscr{D}_{Y \rightarrow X}\right) .
$$

In general, the direct image $f_{+}$is neither right nor left exact. However, if $f$ is an affine morphism, then $f_{*}$ is exact and thus $f_{+}$is right exact. If $\mathscr{D}_{Y \rightarrow X}$ is a flat $\mathscr{D}^{f}$-module, such as when $f$ is an immersion, then the tensor product is exact, so $f_{+}$is left exact. Putting these two special cases together we find that if $f$ is an affine immersion, then $f_{+}$is exact. Moreover, if $f$ is a closed immersion, then $f^{!}$is the right adjoint to $f_{+}$.

Let $f$ be a surjective submersion. In this case, there is a locally free left $\mathscr{D}^{f}$, right $f^{-1} \mathscr{O}_{X}$-module resolution $\mathcal{T}_{Y / X}^{\bullet}\left(\mathscr{D}^{f}\right)$ of $\mathscr{D}_{Y \rightarrow X}$ given by

$$
\mathcal{T}_{Y / X}^{-k}\left(\mathscr{D}^{f}\right)=\mathscr{D}^{f} \otimes_{\mathscr{O}_{Y}} \wedge^{k} \mathscr{T}_{Y / X}, \quad k \in \mathbb{Z},
$$

with the usual de Rham differential. Here $\mathscr{T}_{Y / X}:=\Omega_{Y / X}^{*}$ is the sheaf of local vector fields tangent to the fibers of $f$. Note $\mathscr{T}_{Y / X} \subset \mathscr{D}^{f}$ since the twist of $\mathscr{D}^{f}$ is trivial along these fibers. The direct image with respect to this resolution gives

$$
\begin{aligned}
f_{+}(\mathscr{V}) & =\mathbf{R} f_{*}\left(\mathscr{V} \otimes \omega_{Y / X} \otimes_{\left(\mathscr{D}^{\circ}\right)^{f}} \mathcal{T}_{Y / X}^{\bullet}\left(\mathscr{D}^{\circ}\right)^{f}\right) \\
& =\mathbf{R} f_{*}\left(\Omega_{Y / X}^{\bullet}\left(\mathscr{D}^{f}\right) \otimes_{\mathscr{D}^{f}} \mathscr{V}\right)\left[d_{Y / X}\right]
\end{aligned}
$$

for all $\mathscr{V} \in \mathcal{M}\left(\mathscr{D}^{f}\right)$, where $\Omega_{Y / X}^{\bullet}\left(\mathscr{D}^{f}\right)$ is the relative de Rham complex tensored with $\mathscr{D}^{f}$. In this case, it is transparent that $f_{+}\left[-d_{Y / X}\right]$ is the right adjoint of $f^{\circ}$. 
2.4. Homogeneous twisted sheaves of differential operators. In this section we classify homogeneous sheaves of twisted differential operators on generalized flag varieties. The content follows the analogous constructions in M2] The generalized flag varieties are homogeneous spaces $X$ for a complex reductive group $G$. We consider only tdo's which are equivariant with respect to the $G$-action on $X$; more precisely, we will work exclusively with homogeneous twisted sheaves of differential operators.

Definition 2.2. A homogeneous tdo on a complex $G$-variety $X$ is a tdo $\mathscr{D}$ with a $G$ equivariant structure $\gamma$ and a morphism of algebras $\alpha: \mathcal{U}(\mathfrak{g}) \rightarrow \Gamma(X, \mathscr{D})$ satisfying:

(H1) The group $G$ acts on $\mathscr{D}$ by algebra homomorphisms.

(H2) The differential of $\gamma$ agrees with the adjoint action — that is,

$$
d \gamma_{\xi}(T)=[\alpha(\xi), T], \quad \forall \xi \in \mathfrak{g}, T \in \mathscr{D} .
$$

(H3) The map $\alpha$ is $G$-equivariant.

We now classify homogeneous tdo's on a generalized flag variety $X$ of a complex reductive Lie group $G$. Let $\left(\mathfrak{h}, \Sigma, \Sigma^{+}\right)$be the abstract Cartan data for $\mathfrak{g}$ and recall there is a bijection between subsets $\theta$ of the set of simple positive roots in $\Sigma^{+}$and generalized flag varieties $X$. For our fixed $X$, let $\theta$ denote the corresponding subset of simple roots. We will refer to $X$ and the parabolic subalgebras of $\mathfrak{g}$ corresponding to points of $X$ as being "of type $\theta$ ". Define $\mathfrak{h}_{\theta}$ to be the quotient of $\mathfrak{h}$ by the subspace spanned by the coroots dual to $\theta$. We implicitly will always implicitly consider $\mathfrak{h}_{\theta}^{*}$ as a subspace of $\mathfrak{h}^{*}$ from this point on. Then if $x \in X$ is any point and $\mathfrak{p}_{x}$ the parabolic determined by $x$, there is a specialization short exact sequence

$$
0 \rightarrow\left[\mathfrak{p}_{x}, \mathfrak{p}_{x}\right] \rightarrow \mathfrak{p}_{x} \rightarrow \mathfrak{h}_{\theta} \rightarrow 0
$$

Proposition 2.3. The space $\mathfrak{h}_{\theta}^{*}$ parameterizes isomorphism classes of homogeneous tdo's on the partial flag variety $X$ of type $\theta$.

This proposition is a special case of [M2, Theorem 1.2.4]. The proof is constructive; for completeness, we outline the construction of the homogeneous tdo $\mathscr{D}_{X, \lambda}$ for any $\lambda \in \mathfrak{h}_{\theta}^{*}$. Let $\mathfrak{g}^{\circ}$ denote the trivial bundle $\mathscr{O}_{X} \otimes_{\mathbb{C}} \mathfrak{g}$. There is a surjection $\mathfrak{g}^{\circ} \rightarrow \mathscr{T}_{X}$ with kernel $\mathfrak{p}^{\circ}$, which has geometric fiber $T_{x} \mathfrak{p}^{\circ}=\mathfrak{p}_{x}$ at $x \in X$, where $\mathfrak{p}_{x}$ is the parabolic corresponding to $x$. Let $P_{x}$ be the stabilizer of $x$ in $G$ so that $P_{x}$ has $\mathfrak{p}_{x}$ as its Lie algebra. The specialization $\mathfrak{p}_{x} \rightarrow \mathfrak{h}_{\theta}$ induces an equivalence between $\mathfrak{h}_{\theta}^{*}$ and $P_{x}$-invariant elements of $\mathfrak{p}_{x}^{*}$, so we use $\lambda$ to denote an element of either set. Any $P_{x}$-invariant element $\lambda \in \mathfrak{p}_{x}^{*}$ determines a $G$-equivariant morphism $\lambda^{\circ}: \mathfrak{p}^{\circ} \rightarrow \mathscr{O}_{X}$. Define $\mathcal{U}^{\circ}:=\mathscr{O}_{X} \otimes_{\mathbb{C}} \mathcal{U}(\mathfrak{g})$ and the map $\phi_{\lambda}: \mathfrak{p}^{\circ} \rightarrow \mathcal{U}^{\circ}$ by $\phi_{\lambda}(s)=s-\lambda^{\circ}(s)$ for $s \in \mathfrak{p}^{\circ}$. The image of $\phi_{\lambda}$ generates a two-sided ideal $\mathscr{I}_{\lambda}$ in $\mathcal{U}^{\circ}$; finally, define

$$
\mathscr{D}_{X, \lambda}:=\mathcal{U}^{\circ} / \mathscr{I}_{\lambda}
$$

The action of $G$ on $\mathcal{U}(\mathfrak{g})$ induces an algebraic action on $\mathscr{D}_{X, \lambda}$, and similarly, the surjection $\mathcal{U}^{\circ} \rightarrow \mathscr{D}_{X, \lambda}$ determines a morphism

$$
\alpha: \mathcal{U}(\mathfrak{g}) \rightarrow \Gamma\left(X, \mathscr{D}_{X, \lambda}\right)
$$

upon taking global sections. That the $G$-action and $\alpha$ satisfy (H1)-(H3) is obvious, and therefore, $\mathscr{D}_{X, \lambda}$ is a homogeneous twisted sheaf of differential operators. 
2.5. The infinitesimal character of $\mathscr{D}_{X, \lambda}$. In this section we compute the infinitesimal character of $\Gamma\left(X, \mathscr{D}_{X, \lambda}\right)$. Let $[\lambda] \in \mathfrak{h}^{*} / \mathcal{W}$ be the $\mathcal{W}$-orbit of $\lambda \in \mathfrak{h}^{*}$. Recall that when $X$ is the full flag variety, for any $\lambda \in \mathfrak{h}^{*}$ there is an isomorphism $\Gamma\left(X, \mathscr{D}_{X, \lambda}\right) \simeq \mathcal{U}_{[\lambda-\rho]}$ and all higher cohomology vanishes. Consequently, we define $\mathscr{D}_{\mu}:=\mathscr{D}_{X, \mu+\rho}$ to compensate for the $\rho$-shift in the infinitesimal character of global sections.

Unfortunately, the global sections of $\mathscr{D}_{X, \lambda}$ for $X$ a partial flag variety do not always appear as a quotient of $\mathcal{U}(\mathfrak{g})$. However, we can determine the infinitesimal character without computing global sections explicitly. Define

$$
\mathscr{D}_{\mathfrak{h}_{\theta}}=\mathcal{U}^{\circ} /\left[\mathfrak{p}^{\circ}, \mathfrak{p}^{\circ}\right] \mathcal{U}^{\circ} \text {. }
$$

The quotient $\mathfrak{h}_{\theta}^{\circ}=\mathfrak{p}^{\circ} / D \mathfrak{p}^{\circ}$ is the trivial bundle $\mathfrak{h}_{\theta}^{\circ}=\mathscr{O}_{X} \otimes_{\mathbb{C}} \mathfrak{h}_{\theta}$. For $\lambda \in \mathfrak{h}_{\theta}^{*}$, the corresponding morphism $\phi_{\lambda}: \mathfrak{p}^{\circ} \rightarrow \mathcal{U}^{\circ}$ defining $\mathscr{D}_{X, \lambda}$ descends to $\phi_{\lambda}: \mathfrak{h}_{\theta}^{\circ} \rightarrow \mathscr{D}_{\mathfrak{h}_{\theta}}$. The quotient $\mathfrak{h} \rightarrow \mathfrak{h}_{\theta}$ allows us to extend $\phi_{\lambda}$ to $\mathcal{U}(\mathfrak{h})$, and then compose with the abstract Harish-Chandra isomorphism to get a map $\mathcal{Z}(\mathfrak{g}) \rightarrow \mathscr{D}_{\mathfrak{h}}{ }_{\theta}$. Let $\rho_{\theta}$ and $\rho_{n}$ be defined as in the introduction. Note $\rho_{\theta}$ vanishes in the projection of $\mathfrak{h}^{*}$ to the subspace $\mathfrak{h}_{\theta}^{*}$. Define

$$
\mathscr{D}_{\lambda}=\mathscr{D}_{X, \lambda+\rho_{n}} .
$$

Then, the global sections of the tdo $\mathscr{D}_{\lambda}$ has infinitesimal character $\left[\lambda-\rho_{\theta}\right] \in \mathfrak{h}^{*} / \mathcal{W}$. Hereafter, we use the notation $\mathcal{D}_{[\lambda]}:=\Gamma\left(X, \mathscr{D}_{\lambda}\right)$ for a partial flag variety $X$.

We end with results illustrating some relationships between the twisting parameters for various homogeneous tdo's. Let $p: X \rightarrow X_{\theta}$ be the projection of the full flag variety $X$ to the partial flag variety $X_{\theta}$ of type $\theta$. There is then an equality $\left(\mathscr{D}_{X_{\theta}, \lambda}\right)^{p}=\mathscr{D}_{X, \lambda}$ and so on the full flag variety, we have

$$
\mathscr{D}_{\lambda}^{p}=\mathscr{D}_{\lambda-\rho_{\theta}}=\mathscr{D}_{X, \lambda+\rho_{n}} .
$$

Furthermore, in taking global sections, we have a morphism $\mathcal{U}_{\left[\lambda-\rho_{\theta}\right]} \rightarrow \mathcal{D}_{[\lambda]}$. We may write $\mathcal{U}_{[\lambda]}^{p}$ instead of $\mathcal{U}_{\left[\lambda-\rho_{\theta}\right]}$ for the sake of brevity. Also, since the opposite tdo appears in the construction of the direct image, we include the following proposition.

Proposition 2.4. Let $\mathscr{D}_{\lambda}$ be any homogeneous tdo on the partial flag variety $X_{\theta}$. Then,

$$
\mathscr{D}_{\lambda}^{\circ}=\mathscr{D}_{-\lambda} \cdot
$$

Equivalently, we have $\mathscr{D}_{X_{\theta}, \lambda}^{\circ}=\mathscr{D}_{X_{\theta},-\lambda+2 \rho_{n}}$.

2.6. Anti-dominance and $\mathscr{D}$-affineness. In this section we give some vanishing results for cohomology of $\mathscr{D}$-modules on generalized flag varieties. We use $\Sigma^{\vee}$ to denote the co-roots in $\mathfrak{h}$. For $\lambda \in \mathfrak{h}^{*}$, we say $\lambda$ is anti-dominant if $\alpha^{\vee}(\lambda)$ is not a positive integer for all $\alpha \in \Sigma^{+}$. Further, we say $\lambda$ is regular if the $\alpha^{\vee}(\lambda)$ are all non-zero as well. For $\theta$ a subset of simple roots, let $\Sigma_{\theta}^{+}$denote the closure of $\theta$ in $\Sigma^{+}$under addition. Define $\Sigma_{n}:=\Sigma^{+} \backslash \Sigma_{\theta}^{+}$. For $\mathfrak{p}$ a parabolic of type $\theta$, any specialization of $\left(\mathfrak{h}, \Sigma, \Sigma^{+}\right)$to a Cartan triple for $\mathfrak{p}$ will send $\Sigma_{\theta}^{+}$to positive roots contained in a Levi factor of $\mathfrak{p}$ and $\Sigma_{n}$ to the roots of the nilradical of $\mathfrak{p}$. Let $\rho_{\theta}$ and $\rho_{n}$ be the half-sum of positive roots in $\Sigma_{\theta}^{+}$, respectively, $\Sigma_{n}$. Since $\mathfrak{h}_{\theta}^{*}$ naturally embeds to a subspace of $\mathfrak{h}^{*}$, we can define anti-dominance on $\mathfrak{h}_{\theta}^{*}$ by restricting the condition on $\mathfrak{h}^{*}$. However, due to the shift appearing in (4), and thus in the infinitesimal character of the modules obtained by taking cohomology, it will be more useful to include a shift in the definition. 
Definition 2.5. The character $\lambda \in \mathfrak{h}_{\theta}^{*}$ is anti-dominant (or regular) if $\lambda-\rho_{\theta} \in \mathfrak{h}^{*}$ is.

If $\theta$ is empty, $\mathfrak{h}_{\theta}=\mathfrak{h}$ and $\rho_{\theta}=0$, so this generalized definition for the full flag variety is the usual one. From [BB1, we have the following definition and results.

Definition 2.6. Let $X$ be a generalized flag variety and $\mathscr{D}$ a tdo on $X$. Say $X$ is $\mathscr{D}$-affine if for every $\mathscr{F} \in \mathcal{M}(\mathscr{D})$ we have $\Gamma(X, \mathscr{F})$ generated by global sections and $\mathrm{H}^{i}(X, \mathscr{F})=0$ for all $i>0$.

Proposition 2.7. If $X$ is $\mathscr{D}$-affine, the global sections functor

$$
\Gamma: \mathcal{M}(\mathscr{D}) \rightarrow \mathcal{M}(\mathcal{D})
$$

is an equivalence of categories, where $\mathcal{D}=\Gamma(X, \mathscr{D})$.

Anti-dominance of $\lambda$ is necessary for $\mathscr{D}_{\lambda}$-affineness of the full flag variety $X$; see for example [M3] or BB1]. Note our convention of positive roots is the opposite of [BB1]; i.e., for them dominance rather than anti-dominance of $\lambda$ determines $\mathscr{D}_{\lambda}$-affineness.

Theorem 2.8. Let $X$ be the full flag variety, $\lambda \in \mathfrak{h}^{*}$.

(1) If $\lambda$ is dominant, then $\Gamma: \mathcal{M}\left(\mathscr{D}_{\lambda}\right) \rightarrow \mathcal{M}\left(\mathcal{U}_{[\lambda]}\right)$ is exact.

(2) If $\lambda$ is also regular, then $\Gamma$ is faithful.

A consequence of this theorem is that for $\lambda$ anti-dominant and regular, the global sections functor $\Gamma$ gives an equivalence of categories. Its quasi-inverse $\Delta_{\lambda}$ sends a $\mathcal{U}_{[\lambda]}$-module $V$ to

$$
\Delta_{\lambda}(V)=\mathscr{D}_{\lambda} \otimes_{\mathcal{U}_{[\lambda]}} V
$$

We prove the following proposition in $\$ 5.1$

Proposition 2.9. Let $\lambda \in \mathfrak{h}_{\theta}^{*}$ be anti-dominant and regular. Then $X_{\theta}$ is $\mathscr{D}_{\lambda}$-affine.

\section{THE EQUIVARIANT ZuCKERMAN FUnCTOR}

In this section, we recall the main definitions and some results of the thesis of Pandžić $[\mathrm{P}]$, including the construction of the equivariant Zuckerman functor.

3.1. $(\mathcal{A}, K)$-modules. Let $(\mathcal{A}, K)$ be a pair consisting of an associative algebra $\mathcal{A}$ over $\mathbb{C}$ and $K$ a complex algebraic group. The algebra $\mathcal{A}$ is equipped with an algebraic $K$-action $\phi$, and a $K$-equivariant Lie algebra morphism $\psi: \mathfrak{k} \rightarrow \mathcal{A}$ such that

$$
d \phi(\xi)(a)=[\psi(\xi), a], \quad \xi \in \mathfrak{k}, a \in \mathcal{A} .
$$

Such pairs are called Harish-Chandra pairs. We will eventually take $\mathcal{A}$ to be global sections of a tdo on a generalized flag variety.

Definition 3.1. A weak $(\mathcal{A}, K)$-module is a triple $(V, \pi, \nu)$ consisting of

(1) $V$ an $\mathcal{A}$-module with action $\pi$, and

(2) $V$ an algebraic $K$-module with action $\nu$, such that

(3) the $\mathcal{A}$-action map $\mathcal{A} \otimes V \rightarrow V$ is $K$-equivariant. In other words,

$$
\nu(k) \pi(a) \nu\left(k^{-1}\right)=\pi(\phi(k) a)
$$

for all $k \in K$ and $a \in \mathcal{A}$. 
An $(\mathcal{A}, K)$-module is a weak $(\mathcal{A}, K)$-module such that

(4) $d \nu=\pi \circ \psi$.

Let $\mathcal{M}\left(\mathcal{A}, K^{w}\right)$ be the category of all weak $(\mathcal{A}, K)$-modules. Morphisms of weak $(\mathcal{A}, K)$-modules are linear maps compatible with both the $\mathcal{A}$ - and $K$-module structures. Similarly, denote by $\mathcal{M}(\mathcal{A}, K)$ the category of $(\mathcal{A}, K)$-modules. Let $\mathcal{C}\left(\mathcal{M}\left(\mathcal{A}, K^{(w)}\right)\right), \mathcal{K}\left(\mathcal{M}\left(\mathcal{A}, K^{(w)}\right)\right)$ and $\mathrm{D}\left(\mathcal{M}\left(\mathcal{A}, K^{(w)}\right)\right)$ denote the category of complexes, the homotopy category of complexes, and the derived category of (weak) $(\mathcal{A}, K)$-modules, respectively. For weak modules, we may simplify this notation to $\mathcal{C}\left(\mathcal{A}, K^{w}\right)$, etc., but in the next section we will introduce equivariant categories to replace $\mathcal{C}(\mathcal{M}(\mathcal{A}, K))$, etc.

3.2. Equivariant derived categories. Rather than working in the triangulated categories derived directly from the abelian categories $\mathcal{M}\left(\mathcal{U}_{\chi}, K\right)$ (for some $\chi \in$ $\left.\mathfrak{h}^{*} / \mathcal{W}\right)$, for the purposes of localization it is necessary to work with the equivariant derived category. We give the needed definitions here.

Definition 3.2. An equivariant $(\mathcal{A}, K)$-complex is a pair $\left(V^{\bullet}, i\right)$ with $V^{\bullet}$ a complex of weak $(\mathcal{A}, K)$-modules, and $i$ is a linear map from $\mathfrak{k}$ to graded linear degree -1 endomorphisms of $V^{\bullet}$ satisfying:

(1) The $i_{\xi}$ are $\mathcal{A}$-morphisms for all $\xi \in \mathfrak{k}$.

(2) The $i_{\xi}$ are $K$-equivariant for all $\xi \in \mathfrak{k}$.

(3) The sum $i_{\xi} i_{\eta}+i_{\eta} i_{\xi}=0$ for all $\eta, \xi \in \mathfrak{k}$.

(4) For every $\xi \in \mathfrak{k}$, the sum $d i_{\xi}+i_{\xi} d=\omega(\xi)$ where $\omega=d \nu-\pi \circ \psi$.

We summarize conditions (1) and (2) by stating $i \in \operatorname{Hom}_{K}\left(\mathfrak{k}, \operatorname{Hom}_{\mathcal{A}}\left(V^{\bullet}, V^{\bullet}[-1]\right)\right)$, where we take $\operatorname{Hom}_{\mathcal{A}}\left(V^{\bullet}, V^{\bullet}[-1]\right)$ in the category of graded $\mathcal{A}$-modules, and use the conjugation action of $K$. Specifically, we have $K$ acting on $f \in \operatorname{Hom}_{\mathcal{A}}\left(V^{\bullet}, V^{\bullet}[-1]\right)$ by

$$
(k . f)(v)=\nu(k) \cdot f\left(\nu\left(k^{-1}\right) v\right)
$$

for all $k \in K$ and $v \in V^{\bullet}$. The fourth condition implies the cohomology modules of $V^{\bullet}$ are $(\mathcal{A}, K)$-modules. We will hereafter compress our notation by using $\nu$ to denote both $\nu$ and $d \nu$, and $\pi$ for $\pi$ and $\pi \circ \psi$ so that we may write $\omega=\nu-\pi$. A final notational remark: when referring to a generic equivariant $(\mathcal{A}, K)$-complex $V^{\bullet}$, the associated $(\mathcal{A}, K)$-actions will be denoted $\left(\pi_{V}, \nu_{V}\right)$, and it will also be understood that $\omega_{V}=\nu_{V}-\pi_{V}$.

A morphism of equivariant $(\mathcal{A}, K)$-complexes is a morphism of complexes of weak $(\mathcal{A}, K)$-modules which commutes with $i_{\xi}$ for all $\xi \in \mathfrak{k}$. The category $\mathcal{C}(\mathcal{A}, K)$ of equivariant $(\mathcal{A}, K)$-complexes is abelian. Two morphisms

$$
\phi, \psi:\left(V^{\bullet}, i\right) \rightarrow\left(W^{\bullet}, i\right)
$$

are homotopic if there exists a homotopy of complexes $h: V^{\bullet} \rightarrow W^{\bullet}[-1]$ which anti-commutes with $i_{\xi}$ for all $\xi \in \mathfrak{k}$. That is,

$$
h \circ i_{\xi}=-i_{\xi} \circ h .
$$

Let $\mathcal{K}(\mathcal{A}, K)$ be the homotopy category of equivariant $(\mathcal{A}, K)$-complexes and $\mathrm{D}(\mathcal{A}, K)$ its localization by quasi-isomorphisms. The category $\mathrm{D}(\mathcal{A}, K)$ is known as the equivariant derived category of $(\mathcal{A}, K)$-modules. 
For modules $V^{\bullet}$ and $W^{\bullet} \in \mathcal{C}\left(\mathcal{A}, K^{w}\right)$, define the homomorphism complex (hereafter, "Hom-complex") by setting

$$
\operatorname{Hom}^{k}\left(V^{\bullet}, W^{\bullet}\right)=\prod_{p} \operatorname{Hom}_{\mathcal{M}\left(\mathcal{A}, K^{w}\right)}\left(V^{p}, W^{p+k}\right)
$$

with differential $d^{k}(f)=d_{W} \circ f-(-1)^{k} f \circ d_{V}$. Clearly then $\operatorname{Hom}^{0}\left(V^{\bullet}, W^{\bullet}\right)=$ $\operatorname{Hom}_{\mathcal{C}\left(\mathcal{A}, K^{w}\right)}\left(V^{\bullet}, W^{\bullet}\right)$ and $\mathrm{H}^{0}\left(\operatorname{Hom}^{\bullet}\left(V^{\bullet}, W^{\bullet}\right)\right)=\operatorname{Hom}_{\mathcal{K}\left(\mathcal{A}, K^{w}\right)}\left(V^{\bullet}, W^{\bullet}\right)$. The Homcomplex for objects $\left(V^{\bullet}, i\right)$ and $\left(W^{\bullet}, j\right)$ in $\mathcal{C}(\mathcal{A}, K)$ is defined in the same way, but with morphisms $f \in \operatorname{Hom}^{k}\left(V^{\bullet}, W^{\bullet}\right)$ such that

$$
f i_{\xi}=(-1)^{k} j_{\xi} f, \quad \forall \xi \in \mathfrak{k} .
$$

Again, we have $\operatorname{Hom}^{0}\left(V^{\bullet}, W^{\bullet}\right)=\operatorname{Hom}_{\mathcal{C}(\mathcal{A}, K)}\left(V^{\bullet}, W^{\bullet}\right)$ and $\mathrm{H}^{0}\left(\operatorname{Hom}^{\bullet}\left(V^{\bullet}, W^{\bullet}\right)\right)=$ $\operatorname{Hom}_{\mathcal{K}(\mathcal{A}, K)}\left(V^{\bullet}, W^{\bullet}\right)$.

Let $(*)=\mathcal{C}, \mathcal{K}$ or D. There is a functor For from $_{\mathrm{h}}(*)(\mathcal{A}, K)$ to $(*)\left(\mathcal{A}, K^{w}\right)$ which forgets the homotopy $i$ for the object $\left(V^{\bullet}, i\right)$. Obviously, For ${ }_{\mathrm{h}}: \mathcal{C}(\mathcal{A}, K) \rightarrow$ $\mathcal{C}\left(\mathcal{A}, K^{w}\right)$ is faithful, but the same cannot necessarily be said for the homotopy or derived categories.

For the pair $(\mathfrak{g}, K)$ and $\mathcal{A}=\mathcal{U}(\mathfrak{g})$ (or more generally a quotient of $\mathcal{U}(\mathfrak{g})$ ), an important example of an equivariant complex in $\mathcal{C}^{\mathrm{b}}(\mathcal{A}, K)$ is the standard complex $\mathcal{N}(\mathfrak{g})$ of $\mathfrak{g}$. The complex underlying $\mathcal{N}(\mathfrak{g})$ is the Koszul resolution of $\mathbb{C}$ as a $\mathcal{U}(\mathfrak{g})$ module. That is, for any integer $k$, we have

$$
\mathcal{N}(\mathfrak{g})^{-k}=\mathcal{U}(\mathfrak{g}) \otimes_{\mathbb{C}} \wedge^{k} \mathfrak{g} .
$$

If $u \otimes \tau \in \mathcal{N}(\mathfrak{g})^{-(k+1)}$ with $\tau=\tau_{0} \wedge \ldots \wedge \tau_{k}$, the Koszul differential in degree $-(k+1)$ is

$$
\begin{aligned}
d^{-(k+1)}(u \otimes \tau) & =\sum_{i=0}^{k}(-1)^{i} u \tau_{i} \otimes \tau_{0} \wedge \ldots \hat{\tau}_{i} \ldots \wedge \tau_{k} \\
& +\sum_{0 \leq i<j \leq k}(-1)^{i+j} u \otimes\left[\tau_{i}, \tau_{j}\right] \wedge \tau_{0} \wedge \ldots \hat{\tau}_{i} \ldots \hat{\tau}_{j} \ldots \wedge \tau_{k} .
\end{aligned}
$$

The action $\pi_{N}$ of $\mathfrak{g}$ in any degree is by left multiplication on $\mathcal{U}(\mathfrak{g})$. The action $\nu_{N}$ of $K$ is induced on each side of the tensor product by the map $\phi: K \rightarrow \operatorname{Int}(\mathfrak{g})$, and its differential

$$
d \nu_{N}(\xi)(u \otimes \tau)=d \phi(\xi) u \otimes \tau+u \otimes d \phi(\xi) \tau .
$$

There is a natural homotopy $i$ of these actions on $\mathcal{N}(\mathfrak{g})$ given in the following proposition.

Proposition 3.3. For any $\xi \in \mathfrak{k}, u \otimes \tau \in \mathcal{N}(\mathfrak{g})^{-k}$, define $i_{\xi}(u \otimes \tau)=-u \otimes \psi(\xi) \wedge \tau$. Then, we have $(\mathcal{N}(\mathfrak{g}), i) \in \mathcal{C}^{\mathrm{b}}(\mathcal{A}, K)$.

The proof is a straightforward check, which we omit.

3.3. The right adjoint. The results of $\mathrm{P}$ can be stated in terms of the right adjoint Ind $_{\mathrm{h}}$ to For $_{\mathrm{h}}$ defined below. For the geometric constructions of sections 4 and 5, an alternative definition using tensor products will be more useful. We give both definitions and show they are equivalent.

Define $\operatorname{Ind}_{\mathrm{h}}: \mathcal{C}\left(\mathcal{A}, K^{w}\right) \rightarrow \mathcal{C}(\mathcal{A}, K)$ to take $V^{\bullet} \in \mathcal{C}\left(\mathcal{A}, K^{w}\right)$ to $\operatorname{Ind}_{\mathrm{h}}\left(V^{\bullet}\right)$ with

$$
\operatorname{Ind}_{\mathrm{h}}\left(V^{\bullet}\right)^{i}=\left\{f \in \operatorname{Hom}^{i}\left(\mathcal{N}(\mathfrak{k}), V^{\bullet}\right) \mid f \circ \pi_{N}(\xi)=\omega_{V}(\phi(\xi)) \circ f \forall \xi \in \mathfrak{k}\right\} .
$$

The pair $(\mathcal{A}, K)$ acts on a map $f: \mathcal{N}(\mathfrak{k}) \rightarrow V^{\bullet}$ by

$$
\begin{aligned}
\pi(X) f & =\pi_{V}(X) \circ f, & & \forall X \in \mathcal{A}, \\
\nu(k) f & =\nu_{V}(k) \circ f \circ \nu_{N}\left(k^{-1}\right), & & \forall k \in K,
\end{aligned}
$$


where $\left(\pi_{V}, \nu_{V}\right)$ denote the $(\mathcal{A}, K)$-actions on $V^{\bullet}$. Then, for all $\xi \in \mathfrak{k}$, we have $\omega(\xi) f=-f \circ \omega_{N}(\xi)$. There is a natural homotopy $i$ on $\operatorname{Ind}_{\mathrm{h}}\left(V^{\bullet}\right)$ given by $i_{\xi} f=$ $(-1)^{k-1} f \circ i_{\xi}$ for $f$ a degree $k$ homomorphism, and any $\xi \in \mathfrak{k}$. The following proposition can be proved by direct computation.

Proposition 3.4. For any $V^{\bullet} \in \mathcal{C}\left(\mathcal{A}, K^{w}\right)$, the pair $\left(\operatorname{Ind}_{\mathrm{h}}\left(V^{\bullet}\right), i\right) \in \mathcal{C}(\mathcal{A}, K)$.

That $\mathrm{Ind}_{\mathrm{h}}$ is right adjoint to the forgetful functor $\mathrm{For}_{\mathrm{h}}$ is proved in detail in $\mathrm{K}$, Prop. 4.2.3].

We can construct the right adjoint alternatively using a tensor product. For $V^{\bullet} \in \mathcal{C}\left(\mathcal{A}, K^{w}\right)$, let $\omega_{V}$ denote the $\mathfrak{k}$-action on $V^{\bullet}$ extended to $\mathcal{U}(\mathfrak{k})$. Then, $V^{\bullet}$ can be made into a right $\mathcal{U}(\mathfrak{k})$-module by letting $u \in \mathcal{U}(\mathfrak{k})$ act on $v \in V^{\bullet}$ by $\omega_{V}\left(u^{\iota}\right) v$, where $\iota$ is the principal anti-automorphism of $\mathcal{U}(\mathfrak{k})$. The total tensor product $V^{\bullet} \otimes_{\mathfrak{k}} \mathcal{N}(\mathfrak{k})$ has in degree $k$ the product

$$
\left(V^{\bullet} \otimes_{\mathfrak{k}} \mathcal{N}(\mathfrak{k})\right)^{k}=\prod_{p \in \mathbb{Z}} V^{p} \otimes_{\mathfrak{k}} \mathcal{N}(\mathfrak{k})^{k-p}
$$

and its differential is the usual differential of a double complex

$$
d^{k}(v \otimes n)=d_{V}^{p} v \otimes n+(-1)^{p} v \otimes d_{N}^{k-p} n,
$$

for $v \otimes n \in V^{p} \otimes \mathcal{N}(\mathfrak{k})^{k-p}$. The algebra $\mathcal{A}$ acts on $V^{\bullet} \otimes_{\mathfrak{k}} \mathcal{N}(\mathfrak{k})$ by

$$
\pi(a)(v \otimes n)=\pi_{V}(a) v \otimes n,
$$

for every $a \in \mathcal{A}$ and $v \otimes n \in V^{\bullet} \otimes_{\mathfrak{k}} \mathcal{N}(\mathfrak{k})$. The group $K$ acts diagonally as

$$
\nu(k)(v \otimes n)=\nu_{V}(k) v \otimes \nu_{N}(k) n
$$

for every $k \in K$. Therefore, for every $\xi \in \mathfrak{k}$ we have $\omega(\xi)(v \otimes n)=v \otimes \omega_{N}(\xi) n$. Define the homotopy of actions $i$ to be, up to a sign, the same as for $\mathcal{N}(\mathfrak{k})$. Specifically, for $v \otimes n \in V^{p} \otimes_{\mathfrak{k}} \mathcal{N}(\mathfrak{k})^{k-p}$ and $\xi \in \mathfrak{k}$, let $i_{\xi}(v \otimes n)=(-1)^{p} v \otimes i_{\xi}(n)$.

Proposition 3.5. With the above actions and $i$, the total tensor product $V^{\bullet} \otimes_{\mathfrak{k}} \mathcal{N}(\mathfrak{k})$ is an equivariant $(\mathcal{A}, K)$-complex.

On morphisms, tensoring with $\mathcal{N}(\mathfrak{k})$ sends $f$ to $f \otimes 1$ for every $v \otimes n \in V^{\bullet} \otimes \mathcal{N}(\mathfrak{k})$. One can check that this defines a chain morphism. The construction is the same if we replace $\mathcal{N}(\mathfrak{k})$ by any equivariant $(\mathcal{U}(\mathfrak{k}), K)$-complex, so we have in fact proved the following general theorem.

Theorem 3.6. Let $V^{\bullet} \in \mathcal{C}\left(\mathcal{A}, K^{w}\right)$ and $W^{\bullet} \in \mathcal{C}(\mathcal{U}(\mathfrak{k}), K)$. Take the right $\mathcal{U}(\mathfrak{k})$ module structure on $V^{\bullet}$ determined by $\omega_{V}$. Then $V^{\bullet} \otimes_{\mathfrak{k}} W^{\bullet}$ is in $\mathcal{C}(\mathcal{A}, K)$ with actions defined by

$$
\begin{aligned}
& \pi(a)(v \otimes w)=\pi_{V}(a) v \otimes w \quad \forall a \in \mathcal{A}, \\
& \nu(k)(v \otimes w)=\nu_{V}(k) v \otimes \nu_{W}(k) w \quad \forall k \in K
\end{aligned}
$$

for all $v \otimes w \in V^{p} \otimes W^{k-p}$ and homotopies $i_{\xi}(v \otimes w)=(-1)^{p-1} v \otimes i_{\xi} w$ for all $\xi \in \mathfrak{k}$.

Define $\operatorname{Ind}_{\mathrm{h}}^{\prime}(-):=-\otimes_{\mathcal{U}(\mathfrak{k})} \mathcal{N}(\mathfrak{k})\left[-d_{K}\right]$, where $d_{K}=\operatorname{dim} K$, as a functor from $\mathcal{C}\left(\mathcal{A}, K^{w}\right)$ to $\mathcal{C}(\mathcal{A}, K)$.

Proposition 3.7. The functor $\operatorname{Ind}_{\mathrm{h}}^{\prime}$ is naturally isomorphic to $\operatorname{Ind}_{\mathrm{h}}$. 
Proof. Since $\mathfrak{k}$ is reductive, $\wedge^{d_{K} \mathfrak{k}}=\mathbb{C}$, the trivial representation of $\mathfrak{k}$. Therefore, the natural pairing

$$
\wedge^{p_{\mathfrak{k}}} \times \wedge^{d_{K}-p_{\mathfrak{k}}} \rightarrow \wedge^{d_{K} \mathfrak{k}}
$$

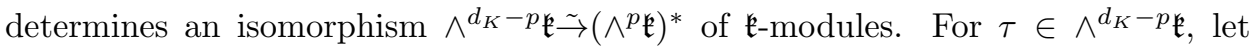
$\tau^{*} \in\left(\wedge^{p} \mathfrak{k}\right)^{*}$ denote the linear map $\tau^{*}(-)=-\wedge \tau$. Then, for any $k \in \mathbb{Z}$, this extends to a vector space isomorphism

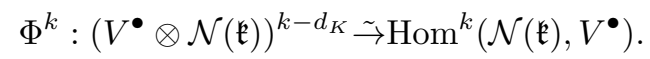

Clearly, the $(\mathcal{A}, K)$-module structure commutes with the vector space isomorphism. Note also, since $(\xi \wedge \tau)^{*}=(-1)^{p+1} \tau^{*} \circ i_{\xi}$, the homotopy $i_{\xi}$ commutes with the

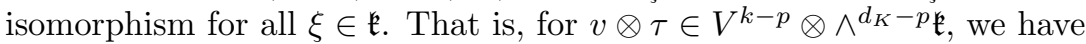

$$
\Phi^{k}\left(i_{\xi}(v \otimes \tau)\right)=i_{\xi}\left(v \otimes \tau^{*}\right) .
$$

The isomorphism $\Phi$ also commutes with differentials. Observe for all $\left(v_{k-p} \otimes\right.$ $\left.\tau_{d_{K}-p}\right) \in \prod_{p} V^{k-p} \otimes \wedge^{d_{K}-p \mathfrak{k}, \text { the compositions }}$

$$
d^{k} \Phi^{k}\left(v_{k-p} \otimes \tau_{d_{K}-p}\right) \quad \text { and } \quad \Phi^{k+1} d^{k-d_{K}}\left(v_{k-p} \otimes \tau_{d_{K}-p}\right)
$$

are equal if and only if

$$
(-1)^{p}\left(d_{N}^{d_{K}-(p-1)} \tau_{d_{K}-(p-1)}\right)^{*}=\tau_{d_{K}-(p-1)}^{*} \circ d_{N}^{p}
$$

for all $p$. Expand $\tau=\tau_{d_{K}-(p-1)}=\xi_{1} \wedge \ldots \wedge \xi_{d_{K}-(p-1)}$ and let $\tau^{\vee}=\zeta_{1} \wedge \ldots \wedge \zeta_{p-1}$ be its complement. For $\zeta \wedge d \tau$ and $d \zeta \wedge \tau$ to be nonzero, (up to sign) either $\zeta=\tau^{\vee} \wedge \xi_{i}$ for some $1 \leq i \leq d_{K}-(p-1)$ or $\zeta=\zeta_{1} \wedge \ldots \hat{\zeta}_{s} \ldots \wedge \zeta_{p-1} \wedge \xi_{i} \wedge \xi_{j}$ for some $1 \leq i<j \leq d_{K}-(p-1)$ and $1 \leq s \leq p-1$. One can verify explicitly that in either of these cases, we do in fact have the equality

$$
(-1)^{p}\left(d_{N}^{d_{K}-(p-1)} \tau\right)^{*}(\zeta)=\tau^{*}\left(d_{N}^{p}(\zeta)\right) .
$$

Therefore, for any $V^{\bullet} \in \mathcal{C}\left(\mathcal{A}, K^{w}\right)$, there is an isomorphism of equivariant $(\mathcal{A}, K)$ complexes

$$
\operatorname{Hom}^{\bullet}\left(\mathcal{N}(\mathfrak{k}), V^{\bullet}\right) \simeq V^{\bullet} \otimes_{\mathfrak{k}} \mathcal{N}(\mathfrak{k})\left[-d_{K}\right]
$$

which is transparently functorial in $V^{\bullet}$.

3.4. Equivariant Zuckerman functor. For $T \subset K$ a closed subgroup, there is a restriction functor $\operatorname{Res}_{T}^{K}$ from $\mathrm{D}(\mathcal{A}, K)$ to $\mathrm{D}(\mathcal{A}, T)$, given simply by restricting the $K$-action on any object to $T$. Pandžić gives an explicit construction of the right adjoint to this functor in $[\mathrm{P}$, which he calls the equivariant Zuckerman functor and denotes by $\Gamma_{K, T}^{\text {equi }}$. We recall the construction here.

Take the standard complex $\mathcal{N}(\mathfrak{k})$ as an object of $\mathcal{C}(\mathfrak{k}, T)$ via the restriction functor. Let $R(K)$ be the ring of regular functions of $K$. Then, for any $V^{\bullet} \in \mathcal{C}(\mathcal{A}, T)$, we have

$$
R(K) \otimes_{\mathbb{C}} V^{\bullet} \in \mathcal{C}(\mathfrak{k}, T)
$$

with the $(\mathfrak{k}, T)$-actions denoted by $\left(\lambda_{\mathfrak{k}}, \lambda_{T}\right)$, respectively. These actions are defined for all $k \in K$ and $F \in R(K) \otimes_{\mathbb{C}} V^{\bullet}$ by

$$
\begin{aligned}
\left(\lambda_{\mathfrak{k}}(\xi) F\right)(k) & =\pi_{V}(\xi) F(k)+L_{\xi} F(k) & & \forall \xi \in \mathfrak{k}, \\
\left(\lambda_{T}(t) F\right)(k) & =\nu_{V}(t) F(k t) & & \forall t \in T,
\end{aligned}
$$


where $\left(\pi_{V}, \nu_{V}\right)$ denote the $(\mathfrak{k}, T)$-actions on $V^{\bullet}$, and the $\mathfrak{k}$-action is extended to $\mathcal{U}(\mathfrak{k})$. The homotopy $i$ is that for $V^{\bullet}$. There is a commuting $(\mathcal{A}, K)$-action on $R(K) \otimes_{\mathbb{C}} V^{\bullet}$, denoted by $\left(\pi_{\Gamma}, \nu_{\Gamma}\right)$ and defined for all $F \in R(K) \otimes V^{\bullet}$ and $k \in K$ by

$$
\begin{array}{lll}
\left(\pi_{\Gamma}(a) F\right)(k)=\pi_{V}(\phi(k) a) F(k), & \forall a \in \mathcal{A}, \\
\left(\nu_{\Gamma}(h) F\right)(k)=F\left(h^{-1} k\right) & & \forall h \in K .
\end{array}
$$

Define the equivariant Zuckerman functor on an object $V^{\bullet} \in \mathcal{C}(\mathcal{A}, T)$ to be

$$
\Gamma_{K, T}^{\text {equi }}\left(V^{\bullet}\right)=\operatorname{Hom}_{\bullet}\left(\mathcal{N}(\mathfrak{k}), R(K) \otimes V^{\bullet}\right)^{(\mathfrak{k}, T)},
$$

with the Hom-complex taken in $\mathcal{C}\left(\mathcal{A}, K^{w}\right)$, and the $(\mathfrak{k}, T)$ indicating that we take $(\mathfrak{k}, T)$-invariants. The $(\mathcal{A}, K)$-action on $\Gamma_{K, T}^{\text {equi }}\left(V^{\bullet}\right)$ is denoted by $(\pi, \nu)$ and defined for all $f \in \Gamma_{K, T}^{\mathrm{equi}}\left(V^{\bullet}\right), n \in \mathcal{N}(\mathfrak{k})$, and $k \in K$ as

$$
\begin{aligned}
& (\pi(a) f)(n)(k)=\left(\pi_{\Gamma}(a) f(n)\right)(k)=\pi_{V}(\phi(k) a) f(n)(k), \quad \forall a \in \mathcal{A}, \\
& (\nu(h) f)(n)(k)=\left(\nu_{\Gamma}(h) f\left(\nu_{N}\left(h^{-1}\right) n\right)\right)(k)=f\left(\nu_{N}\left(h^{-1}\right) n\right)\left(h^{-1} k\right), \quad \forall k^{\prime} \in K .
\end{aligned}
$$

The homotopy $i_{\xi}$ acts on a morphism $f$ in degree $\ell$ by

$$
\left(i_{\xi} f\right)(n)(k)=(-1)^{\ell+1} f\left(i_{\xi} n\right)(k)
$$

for every $n \in \mathcal{N}(\mathfrak{k})$, as in the definition of $\operatorname{Ind}_{\mathrm{h}}$.

\section{Equivariant Harish-Chandra sheaves}

The main technical construction required for the proof of Theorem 1.2 is that of the geometric Zuckerman functor. This is the localization of the equivariant Zuckerman functor to the derived equivariant $\mathscr{D}$-module categories on generalized flag varieties. In this section, we define our categories of interest and construct the geometric Zuckerman functor from the basic $\mathscr{D}$-module functors of $\S 2$.

The category of (left) $G$-equivariant $\mathscr{O}_{X}$-modules is denoted by $\mathcal{M}_{G}(X)$. The following theorem is well known:

Theorem 4.1. If $G$ acts on $X$ freely, there is an equivalence of categories $\mathcal{M}_{G}(X) \simeq \mathcal{M}(X / G)$.

For homogeneous spaces, we can make a stronger statement. If $B$ is a complex linear group, let $\mathcal{R} e p(B)$ be the category of algebraic representations of $B$.

Theorem 4.2. If $X=G / B$, there is an equivalence of categories $\mathcal{M}_{G}(X) \simeq$ $\mathcal{R e p}(B)$.

One realization of this equivalence is by the geometric fiber functor $T_{x}$, where $x \in X$ is the point stabilized by $B$. Recall that the inclusion $i_{x}: x \rightarrow X$ is not $G$-equivariant, but is $B$-equivariant for the restriction of the $G$ action to $B$. Let $\operatorname{Res}_{B}^{G}$ be the functor on $G$-equivariant sheaves which restricts the group action to $B$. Then, define the geometric fiber functor $T_{x}$ to be $i_{x}^{*} \circ \operatorname{Res}_{B}^{G}$. The pull-back $i_{x}^{*}$ is the usual pullback for $\mathscr{O}$-modules. 
4.1. Group and Lie algebra actions on sheaves. Let $e_{k}: X \rightarrow K \times X$ be the map sending $x \mapsto(k, x)$. Then if $\mathscr{F}$ is $K$-equivariant with structure isomorphism

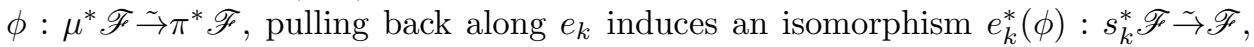
where $s_{k}$ is the automorphism of $X$ given by $x \mapsto k x$. In this way, we map $k \in K$ to the isomorphism

$$
e_{k}^{*}(\phi) \in \prod_{k \in K} \operatorname{Isom}\left(s_{k}^{*}(\mathscr{F}), \mathscr{F}\right),
$$

and thus obtain an action of $K$ on sections. For a local section $f \in \mathscr{F}$, define the action of $k \in K$ on $f$ to be the local section of $\mathscr{F}$ determined by $\nu(k) f:=$ $e_{k}^{*}(\phi)^{-1}(f)$. One differentiates this action to obtain a corresponding Lie algebra action of $\mathfrak{k}$ on the sheaf $\mathscr{F}$. There is a general construction for Lie algebra actions on sheaves given in $\mathrm{BB} 2$.

4.2. Harish-Chandra sheaves. Let $(\mathfrak{g}, K)$ be a Harish-Chandra pair, let $X$ be a generalized flag variety for $\mathfrak{g}$, and let $\mathscr{D}_{\lambda}$ be a homogeneous twisted sheaf of differential operators on $X$. More generally, we can consider Harish-Chandra pairs $(\mathscr{A}, K)$ with $\mathscr{A}$ a sheaf of associative $\mathscr{O}_{X}$-algebras, such that the pair locally satisfies the usual definition of Harish-Chandra pair. A weak Harish-Chandra sheaf for the pair $\left(\mathscr{D}_{\lambda}, K\right)$ is a quasi-coherent $\mathscr{D}_{\lambda}$-module $\mathscr{V}$ with a $K$-equivariant $\mathscr{O}_{X}$-module structure such that the action of $\mathscr{D}_{\lambda}$ is $K$-equivariant. A weak Harish-Chandra sheaf is a Harish-Chandra sheaf if additionally the differential of the $K$-action on $\mathscr{V}$ agrees with the action of $\mathfrak{k}$ induced by $\mathscr{D}_{\lambda}$.

A morphism of weak Harish-Chandra sheaves is a $\mathscr{D}_{\lambda}$-module homomorphism which respects the underlying $K$-equivariant structure. As with weakly equivariant Harish-Chandra modules, we will use $\mathcal{M}\left(\mathscr{D}_{\lambda}, K^{w}\right)$ to denote the category of weak Harish-Chandra sheaves and $\mathcal{M}\left(\mathscr{D}_{\lambda}, K\right)$ for the category of Harish-Chandra sheaves. We construct the derived equivariant Harish-Chandra sheaf category in the same way as the derived equivariant Harish-Chandra module category.

Definition 4.3. An equivariant Harish-Chandra sheaf is a pair $(\mathscr{V} \bullet, i)$ with $\mathscr{V}^{\bullet}$ a complex of weak Harish-Chandra sheaves, and $i$ a linear map from $\mathfrak{k}$ to graded linear degree -1 endomorphisms of $\mathscr{V} \bullet$ satisfying:

(1) The $i_{\xi}$ are $\mathscr{D}_{\lambda}$-morphisms for all $\xi \in \mathfrak{k}$.

(2) The map $i: \mathfrak{k} \rightarrow \operatorname{Hom}_{\mathscr{D}_{\lambda}}(\mathscr{V} \bullet, \mathscr{V} \bullet[-1])$ is $K$-equivariant; that is, for all $k \in K$,

$$
\nu(k) \circ i_{\xi} \circ \nu\left(k^{-1}\right)=i_{\operatorname{Ad}(k) \xi} .
$$

(3) For all $\eta, \xi \in \mathfrak{k}$, the sum $i_{\xi} i_{\eta}+i_{\eta} i_{\xi}$ vanishes.

(4) There is the equality $d i_{\xi}+i_{\xi} d=\omega(\xi)$, where $\omega=\nu-\pi$, where $\nu=d \nu$ and $\pi$ is the action of $\mathfrak{k}$ induced from $\mathscr{D}_{\lambda}$.

Define the Hom-complex $\operatorname{Hom}^{\bullet}(\mathscr{V} \bullet, \mathscr{W} \bullet)$ in the same way as for equivariant Harish-Chandra complexes. The category of equivariant Harish-Chandra sheaves $\mathcal{C}\left(\mathscr{D}_{\lambda}, K\right)$ has equivariant Harish-Chandra sheaves as objects and for any $\mathscr{V} \bullet$ and $\mathscr{W}^{\bullet}$ the morphisms between them are $\operatorname{Hom}^{0}(\mathscr{V} \bullet, \mathscr{W} \bullet)$ as homomorphisms. The homotopy category of equivariant Harish-Chandra sheaves $\mathcal{K}\left(\mathscr{D}_{\lambda}, K\right)$ has the same objects, but the zeroth cohomology $\mathrm{H}^{0}\left(\operatorname{Hom}^{\bullet}(\mathscr{V} \bullet, \mathscr{W} \bullet)\right)$ of the Hom-complex from $\mathscr{V}^{\bullet}$ to $\mathscr{W} \bullet$. The derived equivariant Harish-Chandra sheaf category $\mathrm{D}\left(\mathscr{D}_{\lambda}, K\right)$ is the localization of $\mathcal{K}\left(\mathscr{D}_{\lambda}, K\right)$ with respect to quasi-isomorphisms. According to [MP], 
when $X$ is the full flag variety and $\lambda$ is regular, the derived global sections functor

$$
\mathbf{R} \Gamma: \mathrm{D}^{+}\left(\mathscr{D}_{\lambda}, K\right) \rightarrow \mathrm{D}^{+}\left(\mathcal{U}_{[\lambda]}, K\right),
$$

is an equivalence. This equivariant form of Beilinson-Bernstein localization indicates the equivariant Harish-Chandra sheaf categories are the appropriate geometric category in which to work in the context of the results of $[\mathrm{P}]$.

The categories of interest in the construction of the geometric Zuckerman functor are as follows. Given a generalized flag variety $X$ for a Harish-Chandra pair $(\mathfrak{g}, K)$ and a homogeneous tdo $\mathscr{D}_{\lambda}$ on $X$, we have the categories:

(1) The abelian category $\mathcal{M}\left(\mathscr{D}_{\lambda}, K^{(w)}\right)$ of (weak) Harish-Chandra sheaves on $X$ for the pair $\left(\mathscr{D}_{\lambda}, K\right)$.

(2) The category $\mathcal{C}\left(\mathcal{M}\left(\mathscr{D}_{\lambda}, K^{(w)}\right)\right)$ of complexes of (weak) Harish-Chandra sheaves on $X$, and its homotopy category $\mathcal{K}\left(\mathcal{M}\left(\mathscr{D}_{\lambda}, K^{(w)}\right)\right)$ and derived category $\mathrm{D}\left(\mathcal{M}\left(\mathscr{D}_{\lambda}, K^{(w)}\right)\right)$. For concision, in the case of weak Harish-Chandra sheaves we may abbreviate the above notation to

$$
(*)\left(\mathscr{D}_{\lambda}, K^{w}\right)=(*)\left(\mathcal{M}\left(\mathscr{D}_{\lambda}, K^{w}\right)\right)
$$

for $(*)=\mathcal{C}, \mathcal{K}$, or $\mathrm{D}$.

(3) The category of equivariant Harish-Chandra sheaves for $\left(\mathscr{D}_{\lambda}, K\right)$, denoted $\mathcal{C}\left(\mathscr{D}_{\lambda}, K\right)$ and its homotopy and derived categories $(*)\left(\mathscr{D}_{\lambda}, K\right)$ with $(*)=\mathcal{K}$ and $\mathrm{D}$, respectively.

4.3. The geometric $\operatorname{Ind}_{\mathrm{h}}$. The forgetful functor

$$
\text { For }_{\mathrm{h}}: \mathcal{C}\left(\mathscr{D}_{\lambda}, K\right) \rightarrow \mathcal{C}\left(\mathscr{D}_{\lambda}, K^{w}\right)
$$

forgets the homotopy $i$, as did the forgetful functor for the equivariant $(\mathcal{A}, K)$ complexes of $₫ 3.2$

Proposition 4.4. The forgetful functor $\mathrm{For}_{\mathrm{h}}$ has a right adjoint.

We will now construct a functor $\operatorname{Ind}_{\mathrm{h}}: \mathcal{C}\left(\mathscr{D}_{\lambda}, K^{w}\right) \rightarrow \mathcal{C}\left(\mathscr{D}_{\lambda}, K\right)$ and show in Proposition 4.6 that it is the right adjoint to For $_{\mathrm{h}}$. For an object $\mathscr{V} \bullet \in \mathcal{C}\left(\mathscr{D}_{\lambda}, K^{w}\right)$, put

$$
\operatorname{Ind}_{\mathrm{h}}\left(\mathscr{V}^{\bullet}\right)=\operatorname{Hom}_{\mathcal{U}(\mathfrak{k})}\left(\mathcal{N}(\mathfrak{k}), \mathscr{V}^{\bullet}\right),
$$

where $\mathcal{N}(\mathfrak{k})$ is the standard complex thought of as a constant sheaf on $X$. For any $\xi \in \mathfrak{k}$ define

$$
i_{\xi}: \operatorname{Ind}_{\mathrm{h}}(\mathscr{V} \bullet) \rightarrow \operatorname{Ind}_{\mathrm{h}}(\mathscr{V} \bullet)[-1], \quad i_{\xi} f(u \otimes \tau)=(-1)^{j} f\left(i_{\xi}(u \otimes \tau)\right)
$$

for $f$ in degree $j$, where $i_{\xi}: \mathcal{N}(\mathfrak{k}) \rightarrow \mathcal{N}(\mathfrak{k})[-1]$ is the map

$$
i_{\xi}(u \otimes \tau)=-u \otimes \xi \wedge \tau .
$$

As a $\left(\mathscr{D}_{\lambda}, K\right)$-module, sections of $\mathscr{D}_{\lambda}$ act on $f \in \operatorname{Ind}_{\mathrm{h}}(\mathscr{V} \bullet)$ by their action on $\mathscr{V} \bullet$, and $K$ acts by conjugating $f$ by the $K$-actions on each factor. That is, for any local section $T \in \mathscr{D}_{\lambda}$, we have $(T f)(u \otimes \tau)=T f(u \otimes \tau)$ and

$$
(\nu(k) f)(u \otimes \tau)=\nu_{V}(k) f\left(\nu_{N}\left(k^{-1}\right) u \otimes \tau\right),
$$

where $\nu_{V}$ is the $K$-action on local sections of $\mathscr{V} \bullet$ and $\nu_{N}$ the $K$-action on $\mathcal{N}(\mathfrak{k})$. 
Proposition 4.5. The pair $\left(\operatorname{Ind}_{\mathrm{h}}(\mathscr{V} \bullet), i\right)$ is an object of $\mathcal{C}\left(\mathscr{D}_{\lambda}, K\right)$ for all $\mathscr{V} \bullet \in$ $\mathcal{C}\left(\mathscr{D}_{\lambda}, K^{w}\right)$.

We omit the proof which consists entirely of computations following the definitions.

Proposition 4.6. The functor $\operatorname{Ind}_{\mathrm{h}}$ is right adjoint to For $_{\mathrm{h}}$.

We again omit the completely computational proof, which can be found in $\mathrm{K}$, $\S 5.3]$. A version of this proposition is also presented without proof in BL, Prop. 2.13.2]. The full Hom-complex is used in the proof of adjointness, which allows us to conclude that $\operatorname{Ind}_{\mathrm{h}}$ is also the right adjoint to $\mathrm{For}_{\mathrm{h}}$ for the homotopy category. Moreover, the functor $\mathrm{Ind}_{\mathrm{h}}$ preserves $\mathcal{K}$-injective objects. The proposition below follows immediately from Lemma 4.8 below.

Proposition 4.7. If the category $\mathcal{C}\left(\mathscr{D}_{\lambda}, K^{w}\right)$ has enough $\mathcal{K}$-injectives, then the categories $(*)\left(\mathscr{D}_{\lambda}, K\right)$ for $(*)=\mathcal{C}, \mathcal{K}$ or $\mathrm{D}$ have enough $\mathcal{K}$-injectives.

Since Ind $_{\mathrm{h}}$ is cohomologically finite, this proposition holds for the bounded versions of the involved categories.

Lemma 4.8. Let $(\mathscr{V}, i)$ be an equivariant Harish-Chandra sheaf. If $f: \mathscr{V} \bullet \rightarrow \mathscr{I}^{\bullet}$ is a quasi-isomorphism of $\mathscr{V} \bullet$ with a $\mathcal{K}$-injective $\mathscr{I} \bullet$ in $\mathcal{C}\left(\mathscr{D}_{\lambda}, K^{w}\right)$, then the morphism $\phi_{f} \in \operatorname{Hom}\left(\mathscr{V}^{\bullet}, \operatorname{Ind}_{\mathrm{h}}\left(\mathscr{I}^{\bullet}\right)\right)$ obtained via adjointness is also a quasi-isomorphism.

Proof. Recall from [S, Prop. 1.5] that $\mathscr{I}^{\bullet}$ is $\mathcal{K}$-injective if and only if for all diagrams

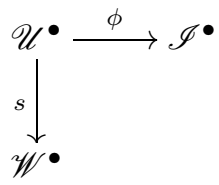

with $s$ a quasi-isomorphism, there exists a unique morphism $g_{\phi}: \mathscr{W}^{\bullet} \rightarrow \mathscr{I}^{\bullet}$ such that $\left[g_{\phi} s\right]=[\phi]$ in the homotopy category.

In the setting of the hypothesis of the lemma, let $\psi$ be a representative of some class $[\psi] \in \mathrm{H}^{k}\left(\operatorname{Ind}_{\mathrm{h}}\left(\mathscr{I}^{\bullet}\right)\right.$, and consider the diagram

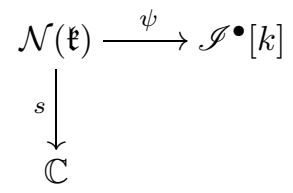

where $s$ is the usual quasi-isomorphism. Then, the unique $g_{\psi}$ which exists such that $\left[g_{\psi} s\right]=[\psi]$ must send 1 to $\psi^{0}(1)$.

Next, let $[v] \in \mathrm{H}^{k}(\mathscr{V} \bullet)$ be the unique element such that $[f(v)]=\left[\psi^{0}(1)\right] \in$ $\mathrm{H}^{k}\left(\mathscr{I}^{\bullet}\right)$. Replacing $\psi$ with $\phi(v)$ in the diagram (6) , we find there exists a unique $g_{f}: \mathbb{C} \rightarrow \mathscr{I} \bullet[k]$ such that $\left[g_{f} s\right]=\left[\phi_{f}(v)\right]$. Therefore,

$$
\left[g_{f}(1)\right]=[f(v)]=\left[g_{\psi}(1)\right] \in \mathrm{H}^{k}(\mathscr{I}) .
$$

This implies $\left[g_{f}\right]=\left[g_{\psi}\right]$, and consequently $\left[\phi_{f}(v)\right]=[\psi]$. 
4.4. Reduction principle. Although the category $\mathrm{D}\left(\mathscr{D}_{\lambda}, K\right)$ is not derived from its heart $\mathcal{M}\left(\mathscr{D}_{\lambda}, K\right)$, the existence of enough $\mathcal{K}$-injectives in $\mathcal{C}\left(\mathscr{D}_{\lambda}, K\right)$ would imply any functor on $\mathcal{C}\left(\mathscr{D}_{\lambda}, K\right)$ which preserves $\mathcal{K}$-injectives can be extended to a functor on $\mathrm{D}\left(\mathscr{D}_{\lambda}, K\right)$. We will show in section 4.6 that $\mathcal{C}^{+}\left(\mathscr{D}_{\lambda}, K^{w}\right)$ has enough $\mathcal{K}$-injectives, which we have seen (Proposition 4.7), implies $\mathcal{C}^{+}\left(\mathscr{D}_{\lambda}, K\right)$ does as well. In fact, the only functors needed in this paper will be cohomologically finite, so we will safely be able to restrict our attention to bounded complexes.

The forgetful functors from $\mathcal{M}\left(\mathscr{D}_{\lambda}, K\right)$ to $\mathcal{M}\left(\mathscr{D}_{\lambda}, K^{w}\right)$ and $\mathcal{C}\left(\mathscr{D}_{\lambda}, K\right)$ to $\mathcal{C}\left(\mathscr{D}_{\lambda}, K^{w}\right)$ are obviously faithful, so any functor on the weakly equivariant categories lifts immediately to Harish-Chandra sheaves and equivariant Harish-Chandra sheaves. The lifting of properties such as exactness, adjointness, etc., follows trivially. It is clear, then, that any exact functor on $\mathcal{M}\left(\mathscr{D}_{\lambda}, K^{w}\right)$ will trivially extend to a functor on $\mathrm{D}\left(\mathscr{D}_{\lambda}, K\right)$. The forgetful functors at the homotopy and derived level are not necessarily faithful, since homotopies of morphisms in the equivariant Harish-Chandra categories must anti-commute with the additional structure map $i$ with which each object is equipped. It is at this point we must rely on the $\mathcal{K}$-injective objects for existence of the equivariant derived functor.

4.5. Functor of $K$-invariants. Lemma 1.8.6 of [BB2] gives an equivalence

$$
\mathcal{M}\left(\mathscr{D}_{\lambda}, K^{w}\right) \simeq \mathcal{M}\left(\mathscr{D}_{\lambda} \otimes_{\mathbb{C}} \mathcal{U}(\mathfrak{k}), K\right) .
$$

The latter category is the category of Harish-Chandra sheaves for the pair $\left(\mathscr{D}_{\lambda} \otimes\right.$ $\mathcal{U}(\mathfrak{k}), K)$. Let $\mathscr{D}$ be any tdo on $X$. Whenever $K$ acts trivially on $X$, there exists a trivial functor $\mathcal{M}(\mathscr{D}) \rightarrow \mathcal{M}(\mathscr{D}, K)$, where the structure morphisms for the HarishChandra pair $(\mathscr{D}, K)$ are the trivial ones; i.e., $\phi(k)=$ id and $\psi(\xi)=0$ for all $k \in K$ and $\xi \in \mathfrak{k}$. Since the action of $K$ on $X$ is trivial, the action and projection morphisms $\mu, \pi: K \times X \rightarrow X$ are equal, hence any $\mathscr{D}$-module on $X$ automatically has the structure of an equivariant $\mathscr{O}_{X}$-module. One can check that this makes our $\mathscr{D}$-module into an $(\mathscr{D}, K)$-module. One can further define the functor $(-)^{K}$ : $\mathcal{M}(\mathscr{D}, K) \rightarrow \mathcal{M}(\mathscr{D})$, which takes $K$-invariant sections of the underlying sheaf. It is exact and right adjoint to the trivial functor.

We must extend the notion of $K$-invariant sections to equivariant Harish-Chandra sheaves. For $K$ acting trivially on $X$, we will from this point on use the notation $(-)^{K}: \mathcal{C}^{\mathrm{b}}\left(\mathscr{D}_{\lambda}, K\right) \rightarrow \mathcal{C}^{\mathrm{b}}\left(\mathscr{D}_{\lambda}\right)$ for the functor taking both $K$ - and $\mathcal{N}(\mathfrak{k})$-invariants. By $\mathcal{N}(\mathfrak{k})$-invariants, we mean $\mathcal{U}(\mathfrak{k})$-invariants which are also invariant for the homotopy map $i$. This invariants functor is right adjoint to the trivial inclusion. Analogously, for an arbitrary $S$-action on $X$, one can define the trivial inclusion $\operatorname{Triv}^{K}:(*)\left(\mathscr{D}_{\lambda}, S^{(w)}\right) \rightarrow(*)\left(\mathscr{D}_{\lambda}, K \times S^{(w)}\right)$ and $(-)^{K}:(*)\left(\mathscr{D}_{\lambda}, K \times S^{(w)}\right) \rightarrow$ $(*)\left(\mathscr{D}_{\lambda}, S^{(w)}\right)$, with $(*)=\mathcal{M}$ or $\mathcal{C}^{\mathrm{b}}$. They are again adjoints.

For arbitrary $K$-action, let $S$ be a closed subgroup of $K$ and define an action of $K \times S$ on $K \times X$ by letting $K$ act on itself on the left and let $S$ act by $s .(k, x)=$ $\left(k s^{-1}, s x\right)$ for all $s \in S$. We refer to this action as the internal diagonal action. When $K$ acts by the given action on $X$ and $S$ acts trivially, the action map $\mu$ : $K \times X \rightarrow X$ is $K \times S$ equivariant. Therefore, the inverse image functor $\mu^{\circ}$ takes (weak) Harish Chandra sheaves on $X$ for the pair $\left(\mathscr{D}_{\lambda}, K \times S\right)$ to (weak) HarishChandra sheaves on $K \times X$. If $K$ acts trivially on $X$ and $S$ acts by the restriction of the given $K$-action, then the projection map $\pi$ is $K \times S$-equivariant. In this case, the functor $\pi_{*}^{K}: \mathcal{M}\left(\mathscr{D}_{\lambda}^{\pi}, K \times S^{(w)}\right) \rightarrow \mathcal{M}\left(\mathscr{D}_{\lambda}, S^{(w)}\right)$ is well defined. 
Recall also [BB2, Lemma 1.8.10], which says that given a free $K$-action on $K \times X$ with $q: K \times X \rightarrow X$ the quotient map, there is then a pair of equivalences

$$
\begin{array}{r}
\mathcal{M}\left(\mathscr{D}_{\lambda}^{q}, K^{w}\right) \underset{q^{\circ}}{\stackrel{q_{*}^{K}}{\rightleftarrows}} \mathcal{M}\left(\mathscr{D}_{\lambda} \otimes \mathcal{U}(\mathfrak{k})\right), \\
\mathcal{M}\left(\mathscr{D}_{\lambda}^{q}, K\right) \underset{q^{\circ}}{\stackrel{q_{*}^{K}}{\rightleftarrows}} \mathcal{M}\left(\mathscr{D}_{\lambda}\right),,
\end{array}
$$

where $q_{*}^{K}$ is the functor of $K$-invariant sections composed with the $\mathscr{O}$-module direct image functor. The inverse image functor $q^{\circ}$ implicitly factors through the trivial inclusion; e.g., $\mathcal{M}\left(\mathscr{D}_{\lambda}\right) \rightarrow \mathcal{M}\left(\mathscr{D}_{\lambda}, K\right)$ for the trivial action of $K$ on $X$ in the latter category.

To extend these equivalences to the equivariant Harish-Chandra categories, we must use the $\mathscr{D}$-module direct image $q_{+}$. The proposition below shows that $q_{+}$ takes equivariant Harish-Chandra sheaves to equivariant Harish-Chandra sheaves.

Proposition 4.9. In the setting of equation (8) , for $(\mathfrak{k}, S)$ a Harish-Chandra pair, if $S$ acts on $K \times X$ and $X$ such that $q$ is $S$-equivariant, then the $\mathscr{D}$-module direct image functor $q_{+}$takes $\mathcal{C}^{\mathrm{b}}\left(\mathscr{D}_{\lambda}^{q}, S\right)$ to $\mathcal{C}^{\mathrm{b}}\left(\mathscr{D}_{\lambda}, S\right)$.

Proof. As it is clear that the functor $q_{+}$lands in $\mathcal{C}^{\mathrm{b}}\left(\mathscr{D}_{\lambda}, S^{w}\right)$, we are left to find a canonical homotopy of the $\mathfrak{s}$-actions

$$
i: \mathfrak{s} \rightarrow \operatorname{Hom}_{\mathscr{D}}\left(q_{+} \mathscr{V}, q_{+} \mathscr{V}[-1]\right)
$$

for every object $(\mathscr{V}, i) \in \mathcal{C}^{\mathrm{b}}\left(\mathscr{D}_{\lambda}^{q}, S\right)$. Since $q$ is a surjective submersion, the direct image functor $q_{+}$is equal to $q_{*}\left(-\otimes_{\mathscr{O}_{K \times X}} \mathcal{T}_{K \times X / X}^{\bullet}\right)$. The sheaf $\mathcal{T}_{K \times X}$ equals the exterior tensor product $\mathcal{T}_{K} \otimes \mathcal{T}_{X}$, and therefore the relative sheaf of differentials is

$$
\mathcal{T}_{K \times X / X} \simeq \pi_{K}^{*} \mathcal{T}_{K}
$$

Moreover, the group $K$ is affine, so $\mathcal{T}_{K}=\mathscr{O}_{K} \otimes_{\mathbb{C}} \mathfrak{k}$ and hence the inverse image is $\pi_{K}^{*} \mathcal{T}_{K}^{\bullet}=\mathscr{O}_{K \times X} \otimes_{\mathbb{C}} \wedge \bullet \mathfrak{k}$. Namely, we have equalities

$$
q_{+}(-)=q_{*}\left(-\otimes_{\mathbb{C}} \wedge \bullet \mathfrak{k}\right)=q_{*}(-) \otimes_{\mathcal{U}(\mathfrak{k})} \mathcal{N}(\mathfrak{k}) .
$$

For any $\mathscr{V} \in \mathcal{C}^{\mathrm{b}}\left(\mathscr{D}_{\lambda}^{q}, S\right)$, the complex $q_{*}(\mathscr{V})$ is a priori only weakly $S$-equivariant. However, since $(\mathfrak{k}, S)$ is Harish-Chandra, the standard complex $\mathcal{N}(\mathfrak{k})$ is an object of $\mathcal{C}^{\mathrm{b}}(\mathcal{U}(\mathfrak{k}), S)$ and therefore we can give $q_{+}(\mathscr{V})$ the structure of an equivariant $\left(\mathscr{D}_{\lambda}, S\right)$-complex by utilizing the homotopy on $\mathcal{N}(\mathfrak{k})$ as in Proposition 3.5.

We will implement this proposition, in particular, for $q=\mu$ or $\pi$ and the group $K \times S$, with the left action of $K$ and the internal diagonal action of $S$ on $K \times X$, where $S \subset K$ is a closed subgroup.

Now we can generalize the equivalence (8) to equivariant Harish-Chandra sheaves. The Triv functor in the corollary below will be used implicitly, and its adjoint $(-)^{K}: \mathcal{C}^{\mathrm{b}}(\mathscr{D}, K) \rightarrow \mathcal{C}^{\mathrm{b}}(\mathscr{D})$ takes both $K$ - and $\mathcal{N}(\mathfrak{k})$-invariants. Note that $\mathcal{N}(\mathfrak{k})$ invariant sections are those invariant for $\mathcal{U}(\mathfrak{k})$ and the homotopy $i$.

Corollary 4.10. For $K$ acting freely on $K \times X$ with quotient map $q: K \times X \rightarrow X$, and for $\mathscr{D}$ a tdo on $X$, there is an equivalence of categories

$$
\mathcal{C}^{\mathrm{b}}\left(\mathscr{D}^{q}, K\right) \underset{q^{\circ}}{\stackrel{q_{+}^{K}\left[-d_{K}\right]}{\rightleftarrows}} \mathcal{C}^{\mathrm{b}}(\mathscr{D}) .
$$


Proof. By the reduction principle, this corollary follows from the equivalence

$$
\mathcal{M}\left(\mathscr{D}^{q}, K^{w}\right) \underset{q^{\circ}}{\stackrel{q_{*}^{K}}{\rightleftarrows}} \mathcal{M}(\mathscr{D} \otimes \mathcal{U}(\mathfrak{k}))
$$

if $q_{+}^{K}\left[-d_{K}\right] \simeq q_{*}^{K}$. Such equivalence follows from the proof of Proposition 4.9. which showed $q_{+}^{K}(-)=\left(q_{*}(-) \otimes_{\mathcal{U}(\mathfrak{k})} \mathcal{N}(\mathfrak{k})\right)^{K}$ with canonical homotopy $i(v \otimes n)=$ $(-1)^{p} v \otimes i n$, where $v$ is of degree $p$. It is then clear the functor $\mathcal{N}(\mathfrak{k})$-invariants composed with $q_{+}$takes the form $q_{*}\left[d_{K}\right]$ and thus $q_{+}^{K}\left[-d_{K}\right] \simeq q_{*}^{K}$.

This equivalence respects (weak) $S$-equivariance when the $S$-action commutes with $K$ and $q$ is $S$-equivariant. That is, this corollary immediately implies an equivalence $\mathcal{C}^{\mathrm{b}}\left(\mathscr{D}^{q}, K \times S^{(w)}\right) \simeq \mathcal{C}^{\mathrm{b}}\left(\mathscr{D}, S^{(w)}\right)$.

4.6. The geometric Zuckerman functor. Let $K$ act on $X$ and let $S \subset K$ be a closed subgroup. Recall for $\mathscr{O}$-modules, there is a restriction functor $\operatorname{Res}_{S}^{K}$ from $\mathcal{M}\left(\mathscr{O}_{X}, K\right)$ to $\mathcal{M}\left(\mathscr{O}_{X}, S\right)$, defined by restricting the $K$-action to $S$. Since the $K$ action on an equivariant sheaf $\mathscr{V}$ is defined by an isomorphism $\phi: \mu^{*} \mathscr{V} \stackrel{\sim}{\rightarrow} \pi^{*} \mathscr{V}$, with $\mu, \pi: K \times X \rightarrow X$ the usual action and projection morphisms (respectively), the restriction of the $K$-action comes from taking $\phi$ to $j^{*} \phi$, where $j: S \times X \rightarrow K \times X$ is the obvious inclusion. One defines the restriction functor on the categories $\mathcal{M}\left(\mathscr{D}_{\lambda}, K^{(w)}\right)$ equivalently. We will use the notation $\operatorname{Res}_{S}^{K}$ for the functor on either category. The functor $\operatorname{Res}_{S}^{K}$ is exact and therefore extends to the equivariant derived categories. For $K$-equivariant Harish-Chandra sheaves, the restriction functor $\operatorname{Res}_{S}^{K}: \mathcal{C}^{\mathrm{b}}\left(\mathscr{D}_{\lambda}, K\right) \rightarrow \mathcal{C}^{\mathrm{b}}\left(\mathscr{D}_{\lambda}, S\right)$ restricts both the $K$-action to $S$ and the map $i$ to $\mathfrak{s}$, the Lie algebra of $S$. We will want another description of this functor, which will be more enlightening for finding its adjoint.

Proposition 4.11. There is a natural isomorphism $\operatorname{Res}_{S}^{K} \simeq \pi_{*}^{K} \mu^{\circ} \operatorname{Triv}^{S}$ as functors from $\mathcal{M}\left(\mathscr{D}_{\lambda}, K\right)$ to $\mathcal{M}\left(\mathscr{D}_{\lambda}, S\right)$.

Proof. For brevity, we drop the Triv from our notation. Let $\mathscr{V} \in \mathcal{M}\left(\mathscr{D}_{\lambda}, K\right)$ be any object, let $\mu_{S}, \pi_{S}: S \times X \rightarrow X$ denote the action and projection morphisms, respectively, let $j$ be as above, and let $e_{S}: X \rightarrow S \times X$ be the identity inclusion $x \mapsto(e, x)$. Forgetting the $\mathscr{D}$-module structure momentarily, note that since $\mu_{S} \circ$ $e_{S}=\pi_{S} \circ e_{S}=i d$, there is an equivalence of underlying $\mathscr{O}$-modules $e_{S}^{*} \mu_{S}^{*} \mathscr{V}=\mathscr{V}$, and likewise for $\pi_{S}$. In particular, we have $\pi_{*}^{K} \mu^{*} \mathscr{V}=e_{S}^{*} \pi_{S}^{*} \pi_{*}^{K} \mu^{*} \mathscr{V}$, but $\pi_{S}=\pi \circ j$, so the right-hand side is isomorphic to $e_{K}^{*} \mu^{*} \mathscr{V}=\mathscr{V}$, where $e_{K}=j \circ e_{S}: X \rightarrow K \times X$ is the identity inclusion. In other words, the $\mathscr{O}$-module underlying the equivariant sheaf $\pi_{*}^{K} \mu^{\circ} \mathscr{V}$ is itself $\mathscr{V}$. Additionally, the $\mathscr{D}$-module structure is unchanged by the functor $\pi_{*}^{K} \mu^{\circ}$. Therefore, the identity morphism $\pi_{*}^{K} \mu^{\circ} \mathscr{V} \rightarrow \operatorname{Res}_{S}^{K} \mathscr{V}$ is an isomorphism of Harish-Chandra modules.

As in the algebraic setting, the geometric restriction functor $\operatorname{Res}_{S}^{K}$ has a right adjoint. The remainder of this section is devoted to constructing the geometric Zuckerman functor $\Gamma_{K, S}^{\text {geo }}$ and proving that it is the right adjoint to $\operatorname{Res}_{S}^{K}$, then extending these results to the derived equivariant category.

Take all morphisms and actions as above (including the last paragraph of section 4.5). Since $\mathscr{D}_{\lambda}$ is a $K$-equivariant $\mathscr{O}_{X}$-module on $X$, there is a canonical isomorphism between $\mu^{*} \mathscr{D}_{\lambda}$ and $\pi^{*} \mathscr{D}_{\lambda}$. Consequently, we have an induced isomorphism $\mathscr{D}_{\lambda}^{\mu} \simeq \mathscr{D}_{\lambda}^{\pi}$ of their respective sheaves of differential endomorphisms. Therefore, 
there is a natural isomorphism of categories $\mathcal{M}\left(\mathscr{D}_{\lambda}^{\mu}\right)=\mathcal{M}\left(\mathscr{D}_{\lambda}^{\pi}\right)$ and we will pass between these two categories without comment or additional notation.

We use the equivalence (8) to motivate our construction of the geometric Zuckerman functor.

Definition 4.12. Define the geometric Zuckerman functor from $\mathcal{C}^{\mathrm{b}}\left(\mathscr{D}_{\lambda}, S\right)$ to $\mathcal{C}^{\text {b }}\left(\mathscr{D}_{\lambda}, K\right)$ to be

$$
\Gamma_{K, S}^{\mathrm{geo}}:=\mu_{+}^{S} \pi^{\circ} \circ \operatorname{Triv}^{K}\left[-d_{K}\right] .
$$

By the reduction principle, the geometric Zuckerman functor $\Gamma_{K, S}^{\text {geo }}$ is also defined for derived equivariant categories if $\mathcal{C}^{\mathrm{b}}\left(\mathscr{D}_{\lambda}, S\right)$ has enough $\mathcal{K}$-injective objects.

Proposition 4.13. The geometric Zuckerman functor $\Gamma_{K, S}^{\text {geo }}$ on the equivariant Harish-Chandra sheaf category is right adjoint to the corresponding restriction functor $\operatorname{Res}_{S}^{K}$.

Proof. By Lemma 4.10, we have that $\pi^{\circ} \circ \operatorname{Triv}^{K}$ and $\pi_{+}^{K}\left[-d_{K}\right]$ are quasi-inverse equivalences. Therefore, the adjointness proposed in the proposition depends only on the right adjointness of $\mu_{+}^{S}\left[-d_{K}\right]$ to $\mu^{\circ} \circ \operatorname{Triv}^{S}$, but since $\mu$ is smooth, we know $\mu^{\circ} \dashv \mu_{+}\left[-d_{K}\right]$ is an adjoint pair.

At the level of the abelian categories of Harish-Chandra sheaves, we can take zeroth cohomology to define the geometric Zuckerman functor; i.e., the functor $\mathrm{H}^{0} \circ \Gamma_{K, S}^{\text {geo }}: \mathcal{M}\left(\mathscr{D}_{\lambda}, S\right) \rightarrow \mathcal{M}\left(\mathscr{D}_{\lambda}, K\right)$ is right adjoint to $\operatorname{Res}_{S}^{K}$. Hereafter, we abuse notation and use $\Gamma_{K, S}^{\text {geo }}$ for the geometric Zuckerman functor on the categories of Harish-Chandra and equivariant Harish-Chandra sheaves alike, as the intent should be clear from context.

In the case $S$ is the trivial subgroup of $K$, the restriction functor merely forgets the $K$-action and its right adjoint preserves injective objects. We abbreviate the notation of these functors to $\operatorname{Res}^{K}$ and to $\Gamma_{K}^{\text {geo }}$. We may also consider weakened versions of the restriction and geometric Zuckerman functor in this case, between the categories $\mathcal{C}^{\mathrm{b}}\left(\mathscr{D}_{\lambda}, K^{w}\right)$ and $\mathcal{C}^{\mathrm{b}}\left(\mathscr{D}_{\lambda} \otimes \mathcal{U}(\mathfrak{k})\right)$. Proposition 4.13 holds with the analogous proof, likewise the analogous statements hold for the abelian categories of weak Harish-Chandra sheaves. This adjoint pair on weak Harish-Chandra sheaves is essential for the following proposition:

Proposition 4.14. The category $\mathcal{M}\left(\mathscr{D}_{\lambda}, K^{w}\right)$ has enough injective objects.

Proof. Since $\mathcal{M}\left(\mathscr{D}_{\lambda} \otimes \mathcal{U}(\mathfrak{k})\right)$ has enough injectives, and $\Gamma_{K}^{\text {geo }}$ preserves injectives, it is enough to show the adjointness morphism $\mathscr{V} \rightarrow \Gamma_{K}^{\text {geo }} \operatorname{Res}^{K}(\mathscr{V})$ is injective for any object $\mathscr{V} \in \mathcal{M}\left(\mathscr{D}_{\lambda}, K^{w}\right)$. Injectivity in this case is a property of the underlying $\mathscr{O}$-module morphism, which in this case is the usual adjointness morphism $\mathscr{V} \rightarrow$ $\mu_{*} \mu^{*} \mathscr{V}$. This is known to be injective in general for projections $Y \times U \rightarrow U$ with $Y$ affine.

Corollary 4.15. The bounded below category of complexes $\mathcal{C}^{\mathrm{b}}\left(\mathscr{D}_{\lambda}, K^{w}\right)$ has enough $\mathcal{K}$-injective objects.

4.7. Properties of $\Gamma_{K, S}^{\text {geo }}$. In this section, we use the geometric Zuckerman functor $\Gamma_{K, S}^{\text {geo }}$ to construct standard modules on generalized flag varieties. With the notations of the last section, let $Q$ be a $K$-orbit on $X$ (a generalized flag variety of type $\theta$ ) and let $i: Q \rightarrow X$ be the inclusion. Fix a point $x \in Q$ and let $i_{x}: x \rightarrow Q$ and 
$j_{x}=i \circ i_{x}$ denote the inclusions of $x$ to $Q$ and $X$, respectively. Recall $\mathcal{D}_{[\lambda]}$ denotes the global sections of $\mathscr{D}_{\lambda}$, where $\lambda$ is in $\mathfrak{h}_{\theta}^{*} \subset \mathfrak{h}^{*}$ and $[\lambda]$ is its Weyl group orbit in $\mathfrak{h}^{*} / \mathcal{W}$. Denote the stabilizer of $x$ in $K$ by $S_{x}$.

Proposition 4.16. The diagram below is commutative:

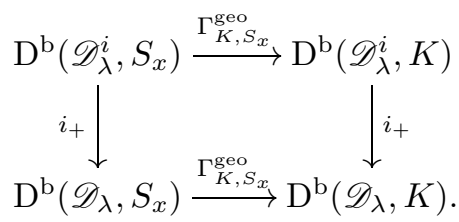

Proof. Consider the fibered square

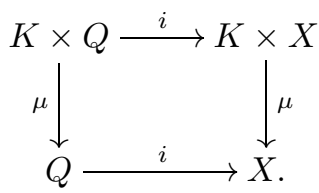

It is clear that $i_{+} \mu_{+}=\mu_{+} i_{+}$. Moreover, we have $i_{+} \mu_{+}^{S_{x}}=\mu_{+}^{S_{x}} i_{+}$, since $i$ is $S_{x^{-}}$ equivariant. A base change for $\mathscr{D}_{\lambda}$-modules allows us to commute $i_{+}$past $\pi^{\circ}$.

Proposition 4.17. The diagram below is commutative:

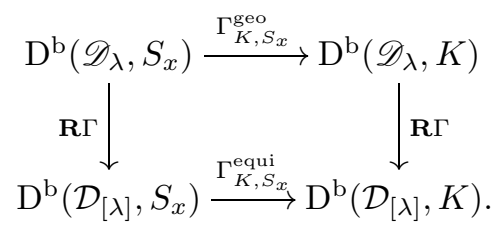

Proof. The reduction principle allows us to work with categories of complexes alone. It is clear from the previous constructions that for any $\mathscr{V}^{\bullet} \in \mathcal{C}^{\mathrm{b}}\left(\mathscr{D}_{\lambda}, S_{x}\right)$, we have

$$
\mathbf{R} \Gamma\left(X, \Gamma_{K, S_{x}}^{\mathrm{geo}} \mathscr{V} \bullet\right)=\operatorname{Hom}_{\left(\mathfrak{k}, S_{x}, \mathcal{N}\left(\mathfrak{s}_{x}\right)\right)}^{\bullet}\left(\mathcal{N}(\mathfrak{k}), \mathbf{R} \Gamma\left(X, \mu_{*} \pi^{\circ} \mathscr{V} \bullet\right)\right)
$$

as $\left(\mathcal{D}_{[\lambda]}, K\right)$-complexes, where $\mathfrak{s}_{x}$ is the Lie algebra of $S_{x}$ and the homomorphisms on the right are equivariant with respect to the $\mathfrak{k}, S_{x}$ and $\mathcal{N}\left(\mathfrak{s}_{x}\right)$-actions. For any open set $U \subset X$, we have by definition $\mu_{*} \pi^{\circ} \mathscr{V} \bullet(U)=R(K) \otimes \mathscr{V} \bullet(U)$ where $R(K)$ is the ring of regular functions on $K$. Therefore, we have

$$
\mathbf{R} \Gamma\left(X, \mu_{*} \pi^{\circ} \mathscr{V}^{\bullet}\right)=R(K) \otimes \mathbf{R} \Gamma\left(X, \mathscr{V}^{\bullet}\right) .
$$

Note that local sections of $\mu_{*} \pi^{*} \mathscr{V} \bullet$ are functions $F$ from $K$ to $\mathscr{V} \bullet$.

The shift isomorphism $\sigma: K \times X \rightarrow K \times X$ (defined by $\sigma(k, x)=(k, k x)$ ) relates $\mu$ and $\pi$ by $\mu=\pi \circ \sigma$. Let $\phi: \mu^{*} \mathscr{D}_{\lambda} \sim \pi^{*} \mathscr{D}_{\lambda}$ be the $K$-equivariance isomorphism, and let $\phi^{*}: \mathscr{D}_{\lambda}^{\mu} \sim_{\rightarrow} \mathscr{D}_{\lambda}^{\pi}$ be the induced isomorphism of tdo's. The isomorphism $\phi^{*}$ locally sends a section $T \in \mathscr{D}_{\lambda}^{\mu}$ to $\phi \circ T \circ \phi^{-1}$, where $\phi: \mu^{*} \mathscr{D}_{\lambda} \stackrel{\sim}{\rightarrow} \pi^{*} \mathscr{D}_{\lambda}$ sends $X \in \mu^{*} \mathscr{D}_{\lambda}$ to $\phi(X)=X \circ \sigma^{-1}$. Now consider the $S_{x}$-action. Let

$$
\tilde{\mu}, \tilde{\pi}: S_{x} \times K \times X \rightarrow K \times X
$$

be the action and projection morphisms, respectively. For any $t \in S_{x}$, let

$$
\tilde{e}_{t}: K \times X \rightarrow S_{x} \times K \times X
$$


be the inclusion $(k, x) \mapsto(t, k, x)$. If $\psi$ is the $S_{x}$-equivariance morphism for $\mathscr{V} \bullet$, then the $S_{x}$-action on $\pi^{*} \mathscr{V} \bullet$ is given by

$$
\lambda_{s}(t)(f \otimes v)=\pi^{*} e_{t}^{*}(\psi)^{-1}(f \otimes v),
$$

where $e_{t}: X \rightarrow S_{x} \times X, e_{t}: x \mapsto(t, x)$. Hence for a local section $F \in \mu_{*} \pi^{*} \mathscr{V} \bullet$, we have

$$
\left(\lambda_{s}(t) F\right)(k)=e_{t}^{*}(\psi)^{-1}(F(k t)) .
$$

The $\mathcal{U}(\mathfrak{k})$-module structure on $\mu_{*} \pi^{*} \mathscr{V} \bullet$ is induced by $\phi^{*}$. For every $F \in \mu_{*} \pi^{*} \mathscr{V}$ and $\xi \in \mathfrak{k}$, we have

$$
\left(\lambda_{\mathfrak{k}}(\xi) F\right)(k)=\phi^{-1}(\xi \phi(F))(k)=L_{\xi} F(k)+\pi_{V}(\xi) F(k) .
$$

On the other hand, $\mathscr{D}_{\lambda}^{\pi}=\mathscr{D}_{K} \otimes \mathscr{D}_{\lambda}$, so for all $T \in \mathscr{D}_{\lambda}$ and $F \in \mu_{*} \pi^{*} \mathscr{V} \bullet$, we have

$$
\left(\pi_{\Gamma}(T) F\right)(k)=\left(\phi^{-1} \circ T \circ \phi\right)(F)(k)=\pi_{V}\left(\nu_{D}\left(k^{-1}\right) T \nu_{D}(k)\right) F(k),
$$

where $\nu_{D}$ denotes the $K$-action on $\mathscr{D}_{\lambda}$, and $\pi_{V}$ the $\mathscr{D}_{\lambda}$-action on $\mathscr{V} \bullet$. Since $\sigma_{k}^{*} \mu_{*} \pi^{*} \mathscr{V} \bullet=\mu_{*} \pi^{*} \mathscr{V} \bullet$ for all $k \in K$, the $K$-action on $F \in \mu_{*} \pi^{*} \mathscr{V} \bullet$ is defined by $\left(\nu_{\Gamma}(k) F\right)\left(k^{\prime}\right)=F\left(k^{-1} k^{\prime}\right)$. The $\left(\mathcal{D}_{\lambda}, K\right)$-actions on $\operatorname{Hom}_{\left(\mathfrak{k}, S_{x}, \mathcal{N}\left(\mathfrak{s}_{x}\right)\right)}(\mathcal{N}(\mathfrak{k})$, $\left.\mathbf{R} \Gamma\left(X, \mu_{*} \pi^{*} \mathscr{V} \bullet\right)\right)$ are given as in the definition of the algebraic functor Ind $\mathrm{h}_{\mathrm{h}}$. Likewise, the homotopy of actions $i$ is defined for a morphism $f: \mathcal{N}(\mathfrak{k}) \rightarrow R(K) \otimes$ $\mathbf{R} \Gamma\left(X, \mathscr{V}^{\bullet}\right)$ of degree $\ell$ by

$$
\left(i_{\xi} f\right)(n)(k)=(-1)^{\ell} f\left(i_{\operatorname{Ad}(k) \xi} n\right)(k)
$$

for all $n \in \mathcal{N}(\mathfrak{k}), k \in K$, where we take the $(\mathcal{U}(\mathfrak{k}), K)$-module structure on $\mathcal{N}(\mathfrak{k})$ from 3.2 Then, by Proposition 4.17, we have an isomorphism

$$
\mathbf{R} \Gamma\left(X, \Gamma_{K, S_{x}}^{\text {geo }}\left(\mathscr{V}^{\bullet}\right)\right) \simeq \Gamma_{K, S_{x}}^{\text {equi }}\left(\mathbf{R} \Gamma\left(X, \mathscr{V}^{\bullet}\right)\right)
$$

of $\left(\mathcal{D}_{[\lambda]}, K\right)$-complexes.

Proposition 4.18. If $\tau$ is a $K$-equivariant connection on $Q$ compatible with $\mathscr{D}^{i}$, then

$$
\Gamma_{K, S_{x}}^{\text {geo }} i_{x+} T_{x} \tau\left[d_{Q}\right] \simeq \tau
$$

Proof. Let $i_{x}: K \rightarrow K \times Q$ be the lift of $i_{x}: x \rightarrow Q$ under the projection $\pi: K \times Q \rightarrow Q$. Denote the quotient and projection maps $q: K \rightarrow Q$ and $p: K \rightarrow x$, respectively, and observe $q=\mu \circ i_{x}$. A base change for $\mathscr{D}$-modules then gives the equivalence

$$
\Gamma_{K, S_{x}}^{\mathrm{geo}} i_{x+}\left[d_{Q}\right]=q_{+}^{S_{x}} \pi^{\circ}\left[-d_{S_{x}}\right] .
$$

Lemma 4.10 provides an equivalence of categories

$$
\mathcal{C}^{\mathrm{b}}\left(\mathscr{D}^{i \circ q}, S_{x}\right) \underset{p_{+}^{K} q^{\circ}\left[-d_{K}\right]}{\stackrel{q_{+}^{S_{x}} p^{\circ}\left[-d_{S_{x}}\right]}{\rightleftarrows}} \mathcal{C}^{\mathrm{b}}\left(\mathscr{D}^{i}, K\right) \text {. }
$$

The corresponding equivalence for the underlying $\mathscr{O}$-module structure is

$$
\mathcal{R} e p\left(S_{x}\right) \underset{p_{*}^{K} q^{*}}{\stackrel{q_{*}^{S_{x}} p^{*}}{\rightleftarrows}} \mathcal{M}\left(\mathscr{O}_{Q}, K\right) .
$$

In this setting, we have $i_{x}^{\circ} \operatorname{Res}_{S_{x}}^{K} \tau=p_{*}^{K} q^{*} \tau=T_{x} \tau$. The equivalence (9) thus implies there is a natural isomorphism $\tau \simeq q_{+}^{S_{x}} \pi^{\circ} T_{x} \tau$. 
Proposition 4.19. With the above notation, if $\phi: X \rightarrow Y$ is a proper $K$ equivariant morphism of projective varieties and $S \subset K$ is any closed subgroup, we have $\phi^{\circ} \Gamma_{K, S}^{\text {geo }}=\Gamma_{K, S}^{\text {geo }} \phi^{\circ}$.

Proof. The inverse image $\phi^{\circ}$ obviously commutes with $\pi^{\circ}$, and it commutes with $\mu_{+}^{S}$ by a base change since $\phi$ is $K$-equivariant, therefore is $S$-equivariant.

\section{Cohomology of Derived Standard modules}

5.1. The Embedding theorem. For a Harish-Chandra pair $(\mathfrak{g}, K)$, let $X_{\theta}$ be a partial flag variety of type $\theta$. Fix a tdo $\mathscr{D}_{\lambda}$ on $X_{\theta}$. Let $X$ denote the full flag variety of $\mathfrak{g}$. There is a natural fibration

$$
p: X \rightarrow X_{\theta}
$$

and a corresponding natural morphism from $\mathcal{U}_{[\lambda]}^{p}=\mathcal{U}_{\left[\lambda-\rho_{\theta}\right]}=\Gamma\left(X, \mathscr{D}_{\lambda}^{p}\right)$ to $\mathcal{D}_{[\lambda]}:=$ $\Gamma\left(X_{\theta}, \mathscr{D}_{\lambda}\right)$. We obtain a pull-back functor

$$
p^{*}: \mathcal{M}\left(\mathcal{D}_{[\lambda]}\right) \rightarrow \mathcal{M}\left(\mathcal{U}_{[\lambda]}^{p}\right)
$$

of modules over these rings in the usual way. The pull-back $p^{*}$ is related to $p^{\circ}$ by the following theorem.

Theorem 5.1 (Embedding Theorem 1.3). The inverse image functor

$$
p^{\circ}: \mathcal{M}\left(\mathscr{D}_{\lambda}\right) \rightarrow \mathcal{M}\left(\mathscr{D}_{\lambda}^{p}\right)
$$

is fully faithful for all $\lambda$, and we have $\Gamma \circ p^{\circ}=p^{*} \circ \Gamma$.

Proof. We will prove full faithfulness of $p^{\circ}$ by constructing a functor from $\mathcal{M}\left(\mathscr{D}_{\lambda}^{p}\right) \rightarrow$ $\mathcal{M}\left(\mathscr{D}_{\lambda}\right)$ which is quasi-inverse to $p^{\circ}$ when restricted to the essential image of $p^{\circ}$. Since $p$ is smooth, the shifted direct image functor $p_{+}[-n]$ is right adjoint to $p^{\circ}$ on the derived categories, where $n$ is the dimension of the fibers of $p$. That is, for any $\mathscr{V} \in \mathcal{M}\left(\mathscr{D}_{\lambda}\right)$ there is a natural morphism of complexes

$$
\text { ad }: \mathscr{V} \rightarrow p_{+} p^{\circ} \mathscr{V}[-n]
$$

As $p$ is a flat morphism between smooth projective varieties, the inverse image $p^{\circ}$ is exact on $\mathcal{M}\left(\mathscr{D}_{\lambda}\right)$. Recall that for surjective submersions the direct image functor $p_{+}$is given by

$$
\mathscr{V} \mapsto \mathbf{R} p_{*}\left(\mathscr{V} \otimes_{\left(\mathscr{D}_{\lambda}^{p}\right)^{\circ}} \mathcal{T}_{X / X_{\theta}}^{\bullet}\left(\mathscr{D}_{\lambda}^{p}\right)^{\circ}\right)=\mathbf{R} p_{*}\left(\mathscr{V} \otimes_{\mathscr{O}_{X}} \omega_{X / X_{\theta}} \otimes_{\left(\mathscr{D}_{\lambda}^{\circ}\right)^{p}} \mathcal{T}_{X / X_{\theta}}^{\bullet}\left(\mathscr{D}_{\lambda}^{\circ}\right)^{p}\right) .
$$

For all the reductions, we will use the first presentation of $p_{+}$, although the left $\mathscr{D}_{\lambda}$-module structure is obscured by this notation.

The relative tangent complex vanishes below the fiber dimension, which implies that for $\mathscr{V} \in \mathcal{M}\left(\mathscr{D}_{\lambda}\right)$ there is a standard truncation triangle

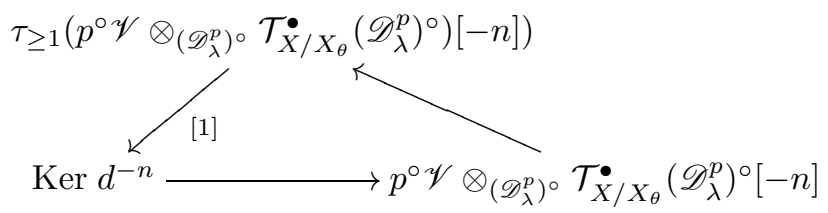

in the derived category, where $d^{\bullet}$ is the differential in $p^{\circ} \mathscr{V} \otimes_{\left(\mathscr{D}_{\lambda}^{p}\right)^{\circ}} \mathcal{T}_{X / X_{\theta}}^{\bullet}$. Since $p_{*}$ is left exact, the long exact sequence obtained from applying $\mathbf{R} p_{*}$ to this triangle induces an isomorphism

$$
p_{*} \operatorname{Ker} d^{-n} \simeq \mathrm{H}^{-n}\left(\mathbf{R} p_{*}\left(p^{\circ} \mathscr{V} \otimes_{\left(\mathscr{D}_{\lambda}^{p}\right)^{\circ}} \mathcal{T}_{X / X_{\theta}}^{\bullet}\left(\mathscr{D}_{\lambda}^{p}\right)^{\circ}\right)\right)
$$


in degree 0 . The adjointness morphism thus descends to cohomology

$$
\mathrm{H}^{0}(\mathrm{ad}): \mathscr{V} \rightarrow \mathbf{R}^{0} p_{*} \operatorname{Ker} d^{-n} .
$$

In fact, since $p_{+}[-n]$ is left exact, the morphism $\mathrm{H}^{0}(\mathrm{ad})$ is injective.

The remainder of the proof proves surjectivity of $\mathrm{H}^{0}(\mathrm{ad})$. The projection formula for $\mathscr{O}$-modules produces the isomorphism

$$
\mathbf{R} p_{*}\left(p^{*} \mathscr{V} \otimes \omega_{X / X_{\theta}} \otimes \mathcal{T}_{X / X_{\theta}}^{\bullet}\right) \simeq \mathscr{V} \otimes \mathbf{R} p_{*}\left(\omega_{X / X_{\theta}} \otimes \mathcal{T}_{X / X_{\theta}}^{\bullet}\right)
$$

it is also an isomorphism of left $\mathscr{D}_{\lambda}$-modules. To see this, we examine the tensor product $\omega_{X / X_{\theta}} \otimes \mathcal{T}_{X / X_{\theta}}$. Define $F_{x}=p^{-1}(p(x))$ and let $\mathfrak{b}_{x}$ be the Borel corresponding to $x$ and similarly $\mathfrak{p}_{x}$ the parabolic corresponding to $p(x)$. There is a short exact sequence

$$
0 \rightarrow \mathfrak{p}_{x} / \mathfrak{b}_{x} \rightarrow \mathfrak{g} / \mathfrak{b}_{x} \rightarrow \mathfrak{g} / \mathfrak{p}_{x} \rightarrow 0
$$

of the tangent spaces. From this sequence we obtain the isomorphisms

$$
T_{x} \mathscr{T}_{X / X_{\theta}}=T_{x} \mathscr{T}_{F_{x}} \simeq \overline{\mathfrak{n}}_{\mathfrak{l}, x} \text { and } T_{x} \Omega_{X / X_{\theta}}=T_{x} \Omega_{F_{x}} \simeq \mathfrak{n}_{\mathfrak{l}, x},
$$

where $\mathfrak{n}_{\mathfrak{l}, x}$ is the nilpotent subalgebra of a Levi factor $\mathfrak{l}_{x}$ of $\mathfrak{p}_{x}$ consisting of positive root spaces of type $\theta$ and $\overline{\mathfrak{n}}_{\mathfrak{l}, x}$ is the opposite nilpotent subalgebra in $\mathfrak{l}_{x}$. In particular, the relative canonical sheaf of $p$ is the homogeneous line bundle

$$
\omega_{X / X_{\theta}}=\mathscr{O}\left(2 \rho_{\theta}\right) \text {. }
$$

Furthermore, since $\mathcal{T}_{X / X_{\theta}}^{-n}=\wedge^{n} \mathscr{T}_{X / X_{\theta}}=\mathscr{O}\left(-2 \rho_{\theta}\right)$, the tensor product $\omega_{X / X_{\theta}} \otimes$ $\mathcal{T}_{X / X_{\theta}}^{-n} \simeq \mathscr{O}_{X}$ is $p_{*}$-acyclic. We claim additionally that for $k \neq n$ we have

$$
\mathbf{R} p_{*}\left(\omega_{X / X_{\theta}} \otimes \mathcal{T}_{X / X_{\theta}}^{k}\right) \simeq 0
$$

Let $U \subset X_{\theta}$ be any open subset trivializing $p$. Then $\left.\mathscr{T}_{X / X_{\theta}}^{-k}\right|_{U \times F}$ is isomorphic to $p_{F}^{*} \wedge^{k} \mathscr{T}_{F}$, where $p_{F}: U \times F \rightarrow F$ is the projection to $F \simeq F_{x}$. Similarly, $\omega_{X / X_{\theta}}=p_{F}^{*} \omega_{F}$. Then, we have

$$
p_{F}^{*}\left(\omega_{F} \otimes \wedge^{k} \mathscr{T}_{F}\right)(U \otimes F)=\mathscr{O}_{X}(U) \otimes \Gamma\left(F, \omega_{F} \otimes \wedge^{k} \mathscr{T}_{F}\right) .
$$

To show (10) holds, it is enough to show $\Gamma\left(F, \omega_{F} \otimes \wedge^{k} \mathscr{T}_{F}\right)$ for all $k \neq n$.

Fix a Levi decomposition $B=H U$, let $\mathfrak{h}$ be the Lie algebra of $H$, and $\mathfrak{n}=[\mathfrak{b}, \mathfrak{b}]$ the maximal nilpotent subalgebra of $\mathfrak{b}$. Note that $\mathfrak{n}$ is the Lie algebra of the unipotent subgroup $U$. Let $\mathfrak{g}_{\alpha}$ be the root space in $\mathfrak{g}$ for root $\alpha$, and $\Delta^{+}$the subset of positive roots. Let $\rho$, as usual, be the half-sum of positive roots.

The sheaf $\omega_{X} \otimes \wedge^{k} \mathscr{T}_{X}$ is the sheaf of sections of the vector bundle $G \times^{B}\left(\mathbb{C}_{2 \rho} \otimes\right.$ $\left.\wedge^{k} \overline{\mathfrak{n}}\right)$, which has a filtration

$$
F_{p}=G \times{ }^{B}\left(\mathbb{C}_{2 \rho} \otimes \wedge^{k} \bar{U}^{p} \overline{\mathfrak{n}}\right),
$$

whose quotients $F_{p} / F_{p+1}$ have as their sheaf of sections

$$
\mathscr{O}(2 \rho) \otimes \bigoplus_{\alpha \in \wedge^{k} \Delta_{p}^{+}} \mathscr{O}(-\alpha),
$$

where $\wedge^{k} \Delta_{p}^{+}$is the set of weights appearing in

$$
\wedge^{k}\left(\bar{U}^{p} \overline{\mathfrak{n}} / \bar{U}^{p+1} \overline{\mathfrak{n}}\right)
$$

That is, it is the set of $k$-fold sums of distinct length $p$ positive roots. Therefore, for $\alpha \in \wedge^{k} \Delta_{p}^{+}$, the difference $2 \rho-\alpha$ is a sum of positive roots, and hence not 
anti-dominant. By the Borel-Weil-Bott theorem, we have $\Gamma(X, \mathscr{O}(2 \rho-\alpha))=0$. It follows that $\Gamma\left(X, \omega_{X} \otimes \wedge^{k} \mathscr{T}_{X}\right)=0$ for all $k \neq n$.

The previous discussion implies that $p_{+} p^{\circ} \mathscr{V}[-n] \simeq \mathscr{V}$. In fact, we proved something stronger:

$$
\left.p_{+}[-n]\right|_{p^{\circ} \mathcal{M}\left(\mathscr{D}_{\lambda}\right)}=\left.p_{*}\right|_{p^{\circ} \mathcal{M}\left(\mathscr{D}_{\lambda}\right)} .
$$

Additionally, the adjointness morphism ad : $\mathscr{V} \rightarrow p_{+} p^{\circ} \mathscr{V}[-n]$ is the identity. Therefore,

$$
\operatorname{Hom}_{\mathscr{D}_{\lambda}^{p}}\left(p^{\circ} \mathscr{V}, p^{\circ} \mathscr{W}\right)=\operatorname{Hom}_{\mathscr{D}_{\lambda}}\left(\mathscr{V}, p_{+} p^{\circ} \mathscr{W}[-n]\right)=\operatorname{Hom}_{\mathscr{D}_{\lambda}}(\mathscr{V}, \mathscr{W}) .
$$

Finally, we address the issue of commutativity with $\mathbf{R} \Gamma$. The equivalence $\mathscr{V} \simeq$ $p_{*} p^{\circ} \mathscr{V}$ implies we have isomorphisms

$$
\mathbf{R} \Gamma\left(X_{\theta}, \mathscr{V}\right)=\mathbf{R} \Gamma\left(X_{\theta}, p_{*} p^{\circ} \mathscr{V}\right) \simeq \mathbf{R} \Gamma\left(X, p^{\circ} \mathscr{V}\right),
$$

of complexes of vector spaces. That the $\mathcal{D}_{[\lambda]}$-actions agree is a consequence of local triviality of the fibration $p: X \rightarrow X_{\theta}$. Locally, the tdo $\mathscr{D}_{\lambda}^{p}=\mathscr{D}_{F} \otimes \mathscr{D}_{\lambda}$ acts on $p^{\circ} \mathscr{V}=\mathscr{O}_{F} \otimes \mathscr{V}$ factorwise. Therefore, the actions of $\mathcal{D}_{[\lambda]}$ and $\mathcal{U}_{[\lambda]}^{p}$ on $\mathbf{R} \Gamma\left(X_{\theta}, \mathscr{V}\right)$ are related by $p^{*}$.

Corollary 5.2. The inverse image functor

$$
p^{\circ}: \mathrm{D}^{\mathrm{b}}\left(\mathscr{D}_{\lambda}, K\right) \rightarrow \mathrm{D}^{\mathrm{b}}\left(\mathscr{D}_{\lambda}^{p}, K\right)
$$

is fully faithful for all $\lambda$, and $\mathbf{R} \Gamma \circ p^{\circ}=p^{*} \circ \mathbf{R} \Gamma$.

Proof. The projection $p$ is $K$-equivariant. Moreover, the proof of the theorem lifts to $\mathcal{M}\left(\mathscr{D}_{\lambda}, K^{w}\right)$. We showed in 4.6 that the adjoint pair $p^{\circ} \dashv \mathbf{R}^{-n} p_{+}$on $\mathcal{M}\left(\mathscr{D}_{\lambda}, K^{w}\right)$ defines an adjoint pair

$$
\mathrm{D}^{\mathrm{b}}\left(\mathscr{D}_{\lambda}, K\right) \underset{p_{+}[-n]}{\stackrel{p^{\circ}}{\rightleftarrows}} \mathrm{D}^{\mathrm{b}}\left(\mathscr{D}_{\lambda}^{p}, K\right) .
$$

Since $p^{\circ}$ is fully faithful on $\mathcal{M}\left(\mathscr{D}_{\lambda}, K^{w}\right)$, the reduction principle implies it is also fully faithful on $\mathrm{D}^{\mathrm{b}}\left(\mathscr{D}_{\lambda}, K\right)$.

The proof of the derived commutation property $\mathbf{R} \Gamma \circ p^{\circ}=p^{*} \circ \mathbf{R} \Gamma$ is the same as the proof for abelian categories.

Corollary 5.3. For $\lambda \in \mathfrak{h}_{\theta}^{*}$ anti-dominant, the functor $\Gamma: \mathcal{M}\left(\mathscr{D}_{\lambda}\right) \rightarrow \mathcal{M}\left(\mathcal{D}_{[\lambda]}\right)$ is exact. If $\lambda$ is also regular, then $\Gamma$ is also faithful.

Proof. Note that $p^{\circ}: \mathcal{M}\left(\mathscr{D}_{\lambda}\right) \rightarrow \mathcal{M}\left(\mathscr{D}_{\lambda}^{p}\right)$, where $\mathscr{D}_{\lambda}^{p}$ is the tdo on the full flag variety corresponding to the parameter $\lambda-\rho_{\theta} \in \mathfrak{h}_{\theta}^{*}$. If $\lambda \in \mathfrak{h}_{\theta}^{*}$ is anti-dominant or regular, then by the definition of these terms for $\mathfrak{h}_{\theta}^{*}$, we have $\lambda-\rho_{\theta} \in \mathfrak{h}^{*}$ is anti-dominant, respectively, regular. Therefore $\Gamma: \mathcal{M}\left(\mathscr{D}_{\lambda}^{p}\right) \rightarrow \mathcal{M}\left(\mathcal{U}_{\left[\lambda-\rho_{\theta}\right]}\right)$ is exact, respectively, faithful. By the embedding theorem, we have $\Gamma \circ p^{\circ}=p^{*} \circ \Gamma$ where $p^{\circ}$ and $p^{*}$ are exact and faithful. The exactness (faithfulness) of $\Gamma: \mathcal{M}\left(\mathscr{D}_{\lambda}\right) \rightarrow \mathcal{M}\left(\mathcal{D}_{[\lambda]}\right)$ then follows from the exactness (faithfulness) of $\Gamma: \mathcal{M}\left(\mathscr{D}_{\lambda}^{p}\right) \rightarrow \mathcal{M}\left(\mathcal{U}_{\left[\lambda-\rho_{\theta}\right]}\right)$.

In the case of integral infinitesimal characters, this result was given by Bien in Bi, Theorem 6.3]. 
5.2. Main Theorem. Let $X_{\theta}$ be the partial flag variety of $\mathfrak{g}$ of type $\theta$. Label the inclusion maps

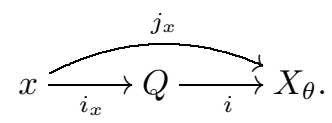

Let $p: X \rightarrow X_{\theta}$ be the natural fibration of the full flag variety $X$ over $X_{\theta}$. From (11) we obtain the following fiber products:

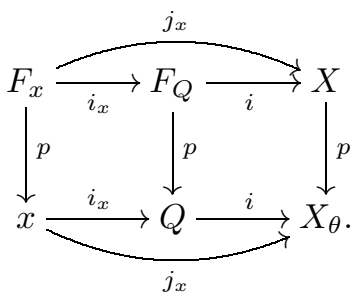

For a fixed point $x \in Q$, let $\mathfrak{p}_{x}$ be the parabolic subalgebra of $\mathfrak{g}$ corresponding to $x$, and let $S_{x}$ be the stabilizer of $x$ in $K$. Let $\mathfrak{n}_{x}$ be the nilpotent subalgebra of $\mathfrak{p}_{x}$. For a $\left(\mathfrak{p}_{x}, S_{x}\right)$-module $Z$, define $Z^{\#}:=Z \otimes \wedge^{d_{X_{\theta}}} \overline{\mathfrak{n}}_{x}$ as a $\left(\mathfrak{p}_{x}, S_{x}\right)$-module via the adjoint action on the twisting factor. For a $\left(\mathfrak{p}_{x}, S_{x}\right)$-module $V$, the induced $\left(\mathfrak{g}, S_{x}\right)$-module is $\operatorname{ind}_{\mathfrak{p}_{x}, S_{x}}^{\mathfrak{g}, S_{x}}(V):=\mathcal{U}(\mathfrak{g}) \otimes_{\mathcal{U}\left(\mathfrak{p}_{x}\right)} V$.

Theorem 5.4 (Main Theorem [1.2). Let $\mathscr{D}_{\lambda}$ be a homogeneous tdo on $X_{\theta}$ and let $\tau$ be a connection on a $K$-orbit $Q$ compatible with $\lambda$. Then there is a quasiisomorphism

$$
\mathbf{R} \Gamma\left(X, p^{\circ} i_{+} \tau\right) \simeq \Gamma_{K, S_{x}}^{\text {equi }}\left(\operatorname{ind}_{\mathfrak{p}_{x}, S_{x}}^{\mathfrak{g}, S_{x}}\left(T_{x} \tau^{\#}\right)\right)\left[d_{Q}\right]
$$

in $\mathrm{D}^{\mathrm{b}}\left(\mathcal{U}_{\left[\lambda-\rho_{\theta}\right]}, K\right)$. Upon taking cohomology, there is a convergent spectral sequence

$$
\mathbf{R}^{p} \Gamma\left(X, p^{\circ} \mathbf{R}^{q} i_{+} \tau\right) \Longrightarrow \mathbf{R}^{p+q+d_{Q}} \Gamma_{K, S_{x}}\left(\operatorname{ind}_{\mathfrak{p}_{x}, S_{x}}^{\mathfrak{g}, S_{x}}\left(T_{x} \tau^{\#}\right)\right) .
$$

Proof. The results of 4.6 define $\Gamma_{K, S_{x}}^{\text {geo }}$ in the general derived equivariant setting and establish the isomorphism $i_{+} \tau \simeq \Gamma_{K, S_{x}}^{\text {geo }} j_{x+} T_{x} \tau\left[d_{Q}\right]$ and commutativity properties (namely $\Gamma_{K, S_{x}}^{\text {geo }} i_{+}=i_{+} \Gamma_{K, S_{x}}^{\text {geo }}, \Gamma_{K, S_{x}}^{\text {geo }} p^{\circ}=p^{\circ} \Gamma_{K, S_{x}}^{\text {geo }}$, and $\mathbf{R} \Gamma \circ \Gamma_{K, S_{x}}^{\text {geo }}=\Gamma_{K, S_{x}}^{\text {equi }} \circ \mathbf{R} \Gamma$ ), which culminate in the natural isomorphisms

$$
\begin{aligned}
\mathbf{R} \Gamma\left(X, p^{\circ} i_{+}(-)\right) & \simeq \mathbf{R} \Gamma\left(X, p^{\circ} \Gamma_{K, S_{x}}^{\text {geo }} j_{x+} T_{x}(-)\right)\left[d_{Q}\right] \\
& \simeq \Gamma_{K, S_{x}}^{\text {equi }} \mathbf{R} \Gamma\left(X, p^{\circ} j_{x+} T_{x}(-)\right)\left[d_{Q}\right] .
\end{aligned}
$$

Then by definition, we have $j_{x+} T_{x} \tau=j_{x *}\left(\mathscr{D}_{X_{\theta} \leftarrow x} \otimes T_{x} \tau\right)$, so

$$
\begin{aligned}
\mathbf{R} \Gamma\left(X, p^{\circ} i_{+} \tau\right) & \simeq \Gamma_{K, S_{x}}^{\text {equi }}\left(\mathscr{D}_{X_{\theta} \leftarrow x} \otimes T_{x} \tau\right)\left[d_{Q}\right] \\
& =\Gamma_{K, S_{x}}^{\text {equi }}\left(\mathcal{U}(\mathfrak{g}) / \mathfrak{p}_{x} \mathcal{U}(\mathfrak{g}) \otimes T_{x} \omega_{X_{\theta}}^{-1} \otimes T_{x} \tau\right)\left[d_{Q}\right]
\end{aligned}
$$

Note that $T_{x} \omega_{X_{\theta}}^{-1}=\wedge^{\text {top }} \overline{\mathfrak{n}}_{x}$ and that the parabolic $\mathfrak{p}_{x}$ acts on $T_{x} \tau^{\#}$ by the $F_{x^{-}}$ invariant linear form $\lambda-\rho_{n}$. Therefore, there is an isomorphism

$$
\mathcal{U}(\mathfrak{g}) / \mathfrak{p}_{x} \mathcal{U}(\mathfrak{g}) \otimes T_{x} \tau^{\#} \simeq \operatorname{ind}_{\mathfrak{p}_{x}, S_{x}}^{\mathfrak{g}, S_{x}}\left(T_{x} \tau^{\#}\right) .
$$

Finally, since $i$ is a locally closed immersion, the direct image $i_{+}$is the composition of the derived $\mathscr{O}_{X}$-module direct image $\mathbf{R} i_{*}$ with an exact functor. The spectral sequence is then seen to follow precisely from the Leray spectral sequence $\mathbf{R}^{p} \Gamma \mathbf{R}^{q} i_{*} \Longrightarrow \mathbf{R}^{p+q}\left(\Gamma \circ i_{*}\right)$. 
When $i_{+}$is exact, the left-hand side of the spectral sequence collapses, and we have the following:

Corollary 5.5. If $i_{+}$is exact, then the following isomorphism holds for all $p$ :

$$
\mathbf{R}^{p} \Gamma\left(X, p^{\circ} i_{+} \tau\right) \simeq \mathbf{R}^{p+d_{Q}} \Gamma_{K, S_{x}}\left(\operatorname{ind}_{\mathfrak{p}_{x}, S_{x}}^{\mathfrak{g}, S_{x}}\left(T_{x} \tau^{\#}\right)\right) .
$$

This is the case for any orbit $Q$ in the full flag variety. Another example is for the open orbit in any partial flag variety of $\mathfrak{g}$ if the Cartan involution defining $K$ is quasi-split. Alternatively, if we are working with twisted differential operators, but take $\lambda$ to be anti-dominant, then $\Gamma$ is exact, and we again find the left-hand side collapses.

Corollary 5.6. For $\lambda$ anti-dominant, we have for all $q$ an isomorphism

$$
\Gamma\left(X, p^{\circ} \mathbf{R}^{q} i_{+} \tau\right) \simeq \mathbf{R}^{q+d_{Q}} \Gamma_{K, S_{x}}\left(\operatorname{ind}_{\mathfrak{p}_{x}, S_{x}}^{\mathfrak{g}, S_{x}}\left(T_{x} \tau^{\#}\right)\right) .
$$

Finally, we can combine the two corollaries to obtain a third:

Corollary 5.7. For $\lambda$ anti-dominant and $i_{+}$exact, we have

$$
\Gamma\left(X, p^{\circ} i_{+} \tau\right) \simeq \mathbf{R}^{d_{Q}} \Gamma_{K, S_{x}}\left(\operatorname{ind}_{\mathfrak{p}_{x}, S_{x}}^{\mathfrak{g}, S_{x}}\left(T_{x} \tau^{\#}\right)\right)
$$

and all other derived Zuckerman functors vanish.

Of possibly the greatest significance is the fact that the convergent spectral sequence (2) determines equalities in the Grothendieck group

$$
\left[\mathbf{R}^{n+d_{Q}} \Gamma_{K, S_{x}}\left(\operatorname{ind}_{\mathfrak{p}_{x}, S_{x}}^{\mathfrak{g}, S_{x}}\left(T_{x} \tau^{\#}\right)\right)\right]=\sum_{p+q=n}\left[\mathbf{R}^{p} \Gamma\left(X, p^{\circ} \mathbf{R}^{q} i_{+} \tau\right)\right]
$$

which may give additional geometric insight into the computation of composition series of degenerate principal series. Low rank examples of applications of this result are discussed in Chapter 8 of $[\mathrm{K}]$.

5.3. Duality and cohomologically induced modules. Fix a Levi subgroup $L_{x}$ of $F_{x}$. Then, $L_{x} \cap K$ is a maximal reductive subgroup of $S_{x}$. The representation $T_{x} \tau$ of the previous section is really a representation of $L_{x} \cap K$ extended trivially to $S_{x}$. For such representations $V$, we have equality of underlying $\mathfrak{g}$-modules

$$
\operatorname{ind}_{\mathfrak{p}_{x}, S_{x}}^{\mathfrak{g}, S_{x}}(V)=\operatorname{ind}_{\mathfrak{p}, L_{x}}^{\mathfrak{g}, L_{x}}(V) .
$$

Abstractly, let $\mathfrak{p} \subset \mathfrak{g}$ be any parabolic, and let $L \subset K$ be a reductive subgroup such that $\mathfrak{l} \subset \mathfrak{p}$. Then, the left adjoint to the forgetful functor from $(\mathfrak{g}, L)$-modules to

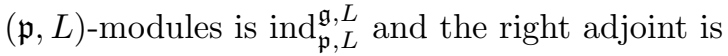

$$
\operatorname{pro}_{\mathfrak{p}, L}^{\mathfrak{g}, L}(-)=\operatorname{Hom}_{\mathfrak{p}}(\mathcal{U}(\mathfrak{g}),-)_{[L]},
$$

where the $[L]$ indicates that we take $L$-finite vectors. For any $(\mathfrak{g}, K)$-module $V$, define the contragredient $V^{\vee}=V_{[K]}^{*}$. Then, we have the following lemma.

Lemma 5.8 ([HMSW], Lemma 3.1). For $V$ any $(\mathfrak{p}, L)$-module,

$$
\operatorname{ind}_{\mathfrak{p}, L}^{\mathfrak{g}, L}(V)^{\vee}=\operatorname{pro}_{\mathfrak{p}, L}^{\mathfrak{g}, L}\left(V^{\vee}\right) .
$$

To identify the modules of Theorem 1.2 as contragredient to cohomologically induced modules, we need to introduce Zuckerman duality: 
Theorem 5.9. Let $G, P$, and $L$ be as above and let $V$ be a $(\mathfrak{l}, L \cap K)$-module. Let $\mathfrak{n}$ be the nilradical of $\mathfrak{p}$ and let $\mathfrak{o}=\mathfrak{k} \cap \mathfrak{n} \oplus \mathfrak{k} \cap \overline{\mathfrak{n}}$ and $s=\operatorname{dim} \mathfrak{k} \cap \mathfrak{n}$. Then, for all $i \geq 0$, there is an isomorphism of $(\mathfrak{g}, K)$-modules

$$
\Gamma_{K, L \cap K}^{i}\left(V^{\vee}\right) \simeq \Gamma_{K, L \cap K}^{2 s-i}\left(\wedge^{\text {top }} \mathfrak{o} \otimes V\right)^{\vee} .
$$

See [HP, Cor. 6.1.9] for the proof of this theorem in the case of real groups. The one-dimensional $(\mathfrak{l}, L \cap K)$-module $\wedge^{\text {top }} \mathfrak{o}$ is trivial for $\mathfrak{l}$, but may have a non-trivial action of the component group of $L \cap K$. Additionally, we have

Theorem 5.10 ( $[\mathrm{MP}$, Theorem 1.13). Let $S \subset K$ be a subgroup and $T$ its Levi factor. The Zuckerman functor $\mathbf{R} \Gamma_{K, S}$ is the restriction of $\mathbf{R} \Gamma_{K, T}$ to $\mathrm{D}(\mathcal{M}(\mathfrak{g}, S))$.

For any $\left(\mathfrak{p}_{x}, L_{x} \cap K\right)$-module $V$, let $V^{\sim}=V \otimes \wedge^{\text {top }} \mathfrak{n}_{x}$. Then, we have $\left(V^{\#}\right)^{\vee}=$ $\left(V^{\vee}\right)^{\sim}$. The $i$ th cohomologically induced module of $V$ is defined to be

$$
\mathcal{R}^{i}(V)=\mathbf{R}^{i} \Gamma_{K, L_{x} \cap K}\left(\operatorname{pro}_{\overline{\mathfrak{p}}_{x}, L_{x} \cap K}^{\mathfrak{g}, L_{x} \cap K}\left(V^{\sim}\right)\right) .
$$

Properties can be found in $\mathrm{HP}$. or [KV]. As a consequence of Theorem 1.2, we have the following corollary.

Corollary 5.11. With the same hypotheses as Theorem 1.2, let $\mathfrak{o}=\mathfrak{k} \cap \mathfrak{n}_{x} \oplus \mathfrak{k} \cap \overline{\mathfrak{n}}_{x}$. Then

$$
\mathbf{R}_{K, S_{x}}^{d_{Q}+i}\left(\operatorname{ind}_{\mathfrak{p}_{x}, L_{x} \cap K}^{\mathfrak{g}, L_{x} \cap K}\left(T_{x} \tau^{\#}\right)\right)^{\vee} \simeq \mathcal{R}^{d_{Q}-i}\left(\left(T_{x} \tau^{\vee} \otimes \wedge^{2 d_{Q}} \mathfrak{o}^{\vee}\right)^{\sim}\right) .
$$

Proof. The duality results yield the isomorphism

$$
\mathbf{R}_{K, S_{x}}^{d_{Q}+i}\left(\operatorname{ind}_{\mathfrak{p}_{x}, L_{x} \cap K}^{\mathfrak{g}, L_{x} \cap K}\left(T_{x} \tau^{\#}\right)\right)^{\vee} \simeq \mathbf{R}^{d_{Q}-i} \Gamma_{K, S_{x}}\left(\operatorname{pro}_{\overline{\mathfrak{p}}_{x}, L_{x} \cap K}^{\mathfrak{g}, L_{x} \cap K}\left(\left(T_{x} \tau^{\#} \otimes \wedge^{2 d_{Q}} \mathfrak{o}\right)^{\vee}\right)\right) .
$$

The observation that $\left(V^{\#}\right)^{\vee}=\left(V^{\vee}\right)^{\sim}$ for any $\left(\mathfrak{p}_{x}, L_{x} \cap K\right)$-module implies

$$
\left(T_{x} \tau^{\#} \otimes \wedge^{2 d_{Q}} \mathfrak{o}\right)^{\vee}=\left(T_{x} \tau^{\vee} \otimes \wedge^{2 d_{Q}} \mathfrak{o}^{\vee}\right)^{\sim},
$$

and Theorem 5.10 completes the proof.

\section{ACKNOWLEDGEMENTS}

I would first like to thank my advisor, Dragan Miličić, without whom none of this would have been possible, and Pavle Pandžić, whose thesis provided the groundwork for mine. Special thanks go to Wolfgang Soergel and Andrew Snowden for all of their useful comments. I would also like to thank the members of the University of Utah math department for their support during my graduate student years and express my gratitude to the NSF, as I was fortunate enough to have been supported by the VIGRE grant for several semesters. Many thanks go also to the referee for his helpful comments, which have greatly improved the clarity and readability of this paper.

\section{REFERENCES}

[BB1] Alexandre Beilinson and Joseph Bernstein, Localisation de g-modules, C. R. Acad. Sci. Paris Ser. I Math. 292 (1981), no. 1, 15-18. MR610137 (82k:14015)

[BB2] - A proof of Jantzen conjectures, I. M. Gel'fand Seminar, Adv. Soviet Math., vol. 16, Amer. Math. Soc., Providence, RI, 1993, pp. 1-50. MR.1237825 (95a:22022)

[BL] Joseph Bernstein and Valery Lunts, Localization for derived categories of $(\mathfrak{g}, K)$-modules, J. Amer. Math. Soc. 8 (1995), no. 4, 819-856. MR1317229 (95m:17004)

[Bi] Fredric Bien, D-modules and spherical representations, Mathematical Notes, vol. 39, Princeton University Press, Princeton, NJ, 1990. MR.1082342 (92f:22025) 
[Bo] A. Borel, P.-P. Grivel, B. Kaup, A. Haefliger, B. Malgrange, and F. Ehlers, Algebraic Dmodules, Perspectives in Mathematics, vol. 2, Academic Press, Inc., Boston, MA, 1987. MR:882000 (89g:32014)

[HMSW] Henryk Hecht, Dragan Miličić, Wilfried Schmid, and Joseph A. Wolf, Localization and standard modules for real semisimple Lie groups I: The duality theorem, Invent. Math. 90 (1987), no. 2, 297-332. MR910203 (89e:22025)

[HP] Jing-Song Huang and Pavle Pandžić, Dirac operators in representation theory, Mathematics: Theory \& Applications, Birkhäuser Boston, Inc., Boston, MA, 2006. MR2244116 (2007j:22025)

[K] S. N. Kitchen, Localization of cohomologically induced modules to partial flag varieties, University of Utah thesis, University of Utah, Salt Lake City, UT, 2010. MR2736734

[KV] Anthony W. Knapp and David A. Vogan, Jr., Cohomological induction and unitary representations, Princeton Matematical Series, vol. 45, Princeton University Press, Princeton, NJ, 1995. MR 1330919 (96c:22023)

[M1] Dragan Miličić, Lectures on algebraic theory of $\mathscr{D}$-modules, Course lecture notes, University of Utah, http://www.math.utah.edu/ milicic.

[M2] - Localization and representation theory of reductive Lie groups, in progress, http://www.math.utah.edu/ milicic.

[M3] - Algebraic $\mathscr{D}$-modules and representation theory of semisimple Lie groups, The Penrose transform and analytic cohomology in representation theory (South Hadley, MA, 1992), Contemp. Math., vol. 154, Amer. Math. Soc., Providence, RI, 1993, pp. 133-168. MR $1246382(94 \mathrm{i}: 22035)$

[MP] Dragan Miličić and Pavle Pandžić, Equivariant derived categories, Zuckerman functors and localization, Geometry and representation theory of real and $p$-adic groups (Cordoba, 1995), Progr. Math., vol. 158, Birkhäuser Boston, Boston, MA, 1998, pp. 209-242. MR:1486143 (2000f:22018)

[P] Pavle Pandžić, Zuckerman functors between equivariant derived categories, Trans. Amer. Math. Soc. 359 (2007), no. 5, 2191-2220 (electronic). MR.2276617(2008a:22016)

[S] N. Spaltenstein, Resolutions of unbounded complexes, Compositio Math. 65 (1988), no. 2, 121-154. MR932640 (89m:18013)

Mathematisches Institut, Albert-Ludwigs-Universität Freiburg, Eckerstr. 1, 79104 Freiburg im Breisgau, Germany

E-mail address: sarah.kitchen@math.uni-freiburg.de 